\title{
Propriedades assintóticas e estimadores consistentes para a probabilidade de clustering
}

\author{
Mariana Pereira de Melo
}

TESE APRESENTADA

$\mathrm{AO}$

Instituto de Matemática E EstatísticA

DA

UNIVERSIDADE DE SÃO PAULO

PARA

OBTENÇÃO DO TÍTULO

$\mathrm{DE}$

DOUTOR EM CIÊNCIAS

Programa: Estatística

Orientador: Prof. Dr. Miguel Natalio Abadi

Durante o desenvolvimento deste trabalho o autor recebeu auxílio financeiro da CAPES/CNPq

São Paulo, 23 de maio de 2014 


\section{Propriedades assintóticas e estimadores consistentes para a probabilidade de clustering}

Esta versão da tese contém as correções e alterações sugeridas pela Comissão Julgadora durante a defesa da versão original do trabalho, realizada em 23/05/2014. Uma cópia da versão original está disponível no Instituto de Matemática e Estatística da Universidade de São Paulo.

Comissão Julgadora:

- Prof. Dr. Miguel Natalio Abadi (orientador) - IME-USP

- Prof. Dr. Cristian Favio Coletti - UFABC

- Prof ${ }^{a}$. Dr ${ }^{a}$. Laura Leticia Ramos Rifo - UNICAMP

- Prof ${ }^{\mathrm{a}}$. Dr ${ }^{\mathrm{a}}$. Veronica Andrea Gonzalez Lopez - UNICAMP

- Prof ${ }^{\mathrm{a}}$. Dr ${ }^{\mathrm{a}}$. Denise Duarte Scarpa Magalhães Alves - UFMG 


\section{Agradecimentos}

Ao meu marido, André, por sua extrema paciência e pelo seu apoio que me conforta e me fortalece para enfrentar novos desafios.

Aos meus familiares que sempre acreditaram e torceram por mim. Em especial, aos meus pais, que me deram não somente a vida, mas principalmente educação e condições de estudo e que, junto com minha irmã, me incentivaram em mais esta etapa da minha vida.

Ao meu orientador, Prof.Dr. Miguel Abadi, por sua orientação, dedicação e incentivo.

Aos membros da Comissão Julgadora por suas observações e sugestões, fundamentais para melhorar este trabalho.

Aos meus amigos, por todas as palavras de incentivo, que me apoiaram nos momentos mais dificeis. Em especial, aos meus amigos Alex, Marina e Núbia que, além de me ensinarem sobre uma cultura diferente, se mostraram pessoas únicas e inesquecíveis.

A todos os colegas que conheci durante a pós-graduação no IME, a todos os funcionários e professores do Instituto.

Meus agradecimentos às entidades financiadoras deste projeto, CAPES e CNPQ, que possibilitaram a realização deste trabalho. 


\section{Resumo}

MELO, M. P. Propriedades assintóticas e estimadores consistentes para a probabilidade de clustering. 2014. 44 f. Tese (Doutorado) - Instituto de Matemática e Estatística, Universidade de São Paulo, São Paulo, 2014.

Considere um processo estocástico $\left(X_{m}\right)_{m \in \mathbb{N}}$ em tempo discreto definido sobre o alfabeto finito $\mathcal{A}$. Seja $x_{0}^{n-1}$ uma palavra fixa sobre $\mathcal{A}^{k}$. No estudo das propriedades estatísticas na teoria de recorrência de Poincaré, é clássico o estudo do tempo decorrente até que a sequência fixa $x_{0}^{k-1}$ seja encontrada em uma realização do processo. Tipicamente, esta é uma quantidade exponencialmente grande com relação ao comprimento da palavra.

Contrariamente, o primeiro tempo de retorno possivel para uma sequência dada está definido como sendo o mínimo entre os tempos de entrada de todas as sequências que começam com a própria palavra e é uma quantidade tipicamente pequena, da ordem do tamanho da palavra.

A convergência quase certa para 1 da razão entre esta quantidade e $k$ é estudada em ([29]) e ([9]). As flutuações desta quantidade foram estudadas em ([5]) e ([24]). Já os grandes desvios desta função foram estudados por ([7]), ([20]) e ([13]).

Neste trabalho estudamos o comportamento da probabilidade deste primeiro retorno possível de uma palavra $x_{0}^{k-1}$ dado que o processo começa com ela mesma. Esta quantidade mede a intensidade de que, uma vez observado um conjunto alvo, possam ser observados agrupamentos ou clusters. Provamos que, sob certas condições, a taxa de decaimento exponencial desta probabilidade converge para a entropia para quase toda a sequência quando k diverge. Apresentamos também um estimador desta probabilidade para árvores de contexto e mostramos sua consistência.

Palavras-chave: tempo de entrada, tempo de retorno, clustering, árvores de contexto. 


\section{Abstract}

MELO, M. P. Asymptotic properties and consistent estimators for the clustering probability. 2014. 44 f. Tese (Doutorado) - Instituto de Matemática e Estatística, Universidade de São Paulo, São Paulo, 2014.

Considering a stochastic process $\left(X_{m}\right)_{m \in \mathbb{N}}$ in a discrete defined time over a finite alphabet $\mathcal{A}$ and $x_{0}^{n-1}$ a fixed word over $\mathcal{A}^{k}$. In the study of the statistical properties of the Poincaré recurrence theory, it is usual the study of the time elapsed until a fixed sequence $x_{0}^{k-1}$ appears in a given realization of process. This quantity is known as the hitting time and it is usually exponentially large in relation to the size of word.

On the opposite, the first possible return time of a given word is defined as the minimum among all the hitting times of realizations that begins with the given word $x_{0}^{k-1}$. This quantity is tipically small that is of the order of the length of the sequence.

The almost surely convergence of the ratio between the first possible return time and $k$ was studied in ([29]) and ([9]). Flutuactions of this quantity were studied in ([5]) and([24]). Large deviations of this function were studied by ([7]), ([20]) and ([12]).

In this work, we study the probability of the first possible return time given that the process begins of the target word. This quantity measures the intensity of that, once observed the target set, it can be observed in clusters. We show that, under certain conditions, the exponential decay rate of this probability converges to the entropy for all almost every word $x_{0}^{k-1}$ as $k$ diverges. We also present an estimator of this probability for context trees and shows its consistency.

Keywords: hitting time, return time, clustering, context trees. 


\section{Sumário}

1 Introdução 1

1.1 Definições básicas . . . . . . . . . . . . . . . . . . . . . . . . . . 1

1.2 Resultados Anteriores . . . . . . . . . . . . . . . . . . . . . . . . 2

1.2.1 Função Primeiro retorno possível . . . . . . . . . . . . . . . . . 2

1.2.2 Função Tempo de Entrada . . . . . . . . . . . . . . . . . . . 3

1.2.3 Função Tempo de retorno . . . . . . . . . . . . . . . . . . . 3

1.2.4 Tipicalidade de larga-escala . . . . . . . . . . . . . . . . . . . 3

1.3 Objetivos e Resultados Obtidos . . . . . . . . . . . . . . . . . . . . . . . 4

2 Propriedades assintóticas $\quad 7$

2.1 Definições ................................... . . 7

2.1.1 Definições relativas à dependência . . . . . . . . . . . . . . . . . . 8

2.1.2 Preliminares............................ 8

2.2 Propriedades assintóticas de $\tilde{\rho}\left(x_{0}^{k-1}\right)$ e $\rho\left(x_{0}^{k-1}\right)$ para processos $\psi_{0}$-regulares. $\quad$. . . . 9

2.3 Propriedades assintóticas de $\tilde{\rho}\left(x_{0}^{k-1}\right)$ e $\rho\left(x_{0}^{k-1}\right)$ para processos $\psi_{g}$-regulares, com $g>0$. 10

2.4 Contra-exemplo: Processo ergódico e de entropia positiva . . . . . . . . . . . . . . . 12

3 Propriedades dos Estimadores $\quad 17$

3.1 Árvores de Contexto . . . . . . . . . . . . . . . . . . . . . . . . . 17

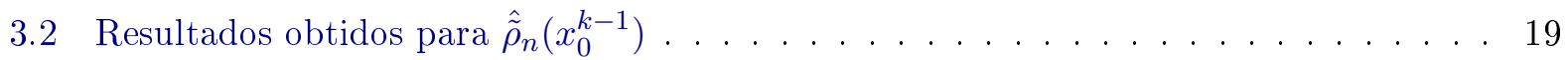

3.2.1 Convergência do estimador $\hat{\tilde{\rho}}_{n}\left(x_{0}^{k-1}\right)$. . . . . . . . . . . . . . . . 25

3.3 Resultados obtidos para $\hat{\rho}_{n}\left(x_{0}^{k-1}\right)$. . . . . . . . . . . . . . . . . . . . . 25

3.3.1 Convergência do estimador $\hat{\rho}_{n}\left(x_{0}^{k-1}\right) \ldots \ldots \ldots \ldots$. . . . . . . . . . . 31

$\begin{array}{lll}\text { A } & \text { Ferramentas auxiliares } & 33\end{array}$

Referências Bibliográficas $\quad 45$ 
viii SUMÁRIO 


\section{Capítulo 1}

\section{Introdução}

\subsection{Definições básicas}

Considere um processo estocástico $\left(X_{m}\right)_{m \in \mathbb{N}}$ em tempo discreto definido sobre o alfabeto finito $\mathcal{A}$. Para cada inteiro positivo $k$, considere o conjunto de todas as palavras de comprimento $k$ sobre este alfabeto, denominado $\mathcal{A}^{k}$.

Definimos primeiramente o tempo de entrada de uma realização $\omega$ do processo em $x_{0}^{k-1} \in \mathcal{A}^{k}$ como

$$
T_{x_{0}^{k-1}}(\omega)=\inf \left\{j \geq 1: X_{j}^{j+k-1}(\omega)=x_{0}^{k-1}\right\} .
$$

Este é o tempo de entrada clássico estudado na teoria de recorrência de Poincaré, que verifica, entre outras coisas, o conhecido Lema de Kac. Tipicamente, esta é uma quantidade de magnitude exponencialmente grande. Porém, neste trabalho consideraremos uma quantidade tipicamente pequena, de fácil observação nas amostras.

Seja a função $\tau\left(x_{0}^{k-1}\right)$ definida como primeiro retorno possível para $x_{0}^{k-1}$, dada por

$$
\tau\left(x_{0}^{k-1}\right)=\inf _{\left\{\omega: w_{0}^{k-1}=x_{0}^{k-1}\right\}} T_{x_{0}^{k-1}}(\omega) .
$$

Mesmo quando a definição de $T_{x_{0}^{k-1}}$ é puramente analítica, é interessante ter em mente uma definição equivalente do ponto de vista combinatorial. Fixada uma palavra de tamanho $k, x_{0}^{k-1}$, o tempo de retorno de $x_{0}^{k-1}$ sobre toda seqüência infinita $\omega$, tal que $w_{0}^{k-1}=x_{0}^{k-1}$, é definido explicitamente como

$$
T_{x_{0}^{k-1}}(\omega)=\inf \left\{j \geq 1: x_{0}^{k-j-1}=x_{j}^{k-1}\right\}
$$

(e infinito caso contrário), onde $\omega$ é uma realização do processo. Então

$$
\tau\left(x_{0}^{k-1}\right)=\inf _{\left\{\omega: w_{0}^{k-1}=x_{0}^{k-1}\right\}} T_{x_{0}^{k-1}}(\omega) .
$$

Para uma palavra $x_{0}^{k-1}$, a função primeiro retorno possível nos dá o número mínimo de translações necessárias que temos que aplicar na sequência, a fim de poder encontrar uma cópia dela mesma.

Considere $\lambda\left(x_{0}^{k-1}\right)$ a probabilidade de saída de $x_{0}^{k-1}$, definida como

$$
\lambda\left(x_{0}^{k-1}\right)=\mathbb{P}\left(T_{x_{0}^{k-1}}(\omega)>\tau\left(x_{0}^{k-1}\right) \mid X_{0}^{k-1}=x_{0}^{k-1}\right)
$$

ou, equivalentemente,

$$
\lambda\left(x_{0}^{k-1}\right)=\mathbb{P}\left(X_{\tau\left(x_{0}^{k-1}\right)}^{\tau\left(x_{0}^{k-1}\right)+k-1} \neq x_{0}^{k-1} \mid X_{0}^{k-1}=x_{0}^{k-1}\right) .
$$


Podemos definir $\rho\left(x_{0}^{k-1}\right)$ a partir da probabilidade de saída,

$$
\rho\left(x_{0}^{k-1}\right)=\mathbb{P}\left(T_{x_{0}^{k-1}}(\omega)=\tau\left(x_{0}^{k-1}\right) \mid X_{0}^{k-1}=x_{0}^{k-1}\right)
$$

ou, equivalentemente,

$$
\rho\left(x_{0}^{k-1}\right)=\mathbb{P}\left(X_{\tau\left(x_{0}^{k-1}\right)}^{\tau\left(x^{k-1}\right)+k-1}=x_{0}^{k-1} \mid X_{0}^{k-1}=x_{0}^{k-1}\right) .
$$

Observe que $\rho\left(x_{0}^{k-1}\right)$ é a probabilidade de permanecer em $x_{0}^{k-1}$, isto é, $\rho\left(x_{0}^{k-1}\right)=1-\lambda\left(x_{0}^{k-1}\right)$. Esta quantidade mede a intensidade de que, uma vez observado um conjunto alvo, possam ser observados agrupamentos ou clusters.

\subsection{Resultados Anteriores}

\subsubsection{Função Primeiro retorno possível}

A relevância da função primeiro retorno possível foi colocada em evidência na análise estatística da recorrência de Poincaré. Para provar a convergência do número de ocorrências de uma palavra de tamanho $k$ em uma amostra de tamanho $n$, quando $k$ diverge, para a distribuição de Poisson é necessário que a palavra não se sobreponha ([22]) ou, pelo menos, que a proporção que sobreponhase, em relação a $k$, seja pequena. Se este não for o caso, uma distribuição de Poisson é a lei limitante ([19]). Há também algumas aproximações para esse limite ([26], [27] e [28]).

Os primeiros trabalhos a estudar o comportamento da função de primeiro retorno foram ([14]) e ([1]), onde é provado que

$$
\mathbb{P}\left(\tau\left(x_{0}^{k-1}\right) \leq c k\right) \leq C \exp ^{-d k},
$$

com $C, c$ e $d$ constantes positivas tal que $c \in[0,1]$. Os resultados foram estabelecidos para processos $\psi$-mixing assumindo decaimento exponencial de $\{\psi(m)\}_{m \in \mathbb{N}}$ e para processos $\phi$-mixing assumindo decaimento exponencial de $\{\phi(m)\}_{m \in \mathbb{N}}$, respectivamente.

Posteriormente, usando a função de complexidade de Kolmogorov e, independentemente, utilizando o Teorema de Shannon, Mc-Millan e Breiman, foi provado em ([29]) e ([9]) respectivamente que, para um processo estocástico sobre um alfabeto finito ou contável, com uma medida ergódica positiva $\mu$ e entropia positiva satisfazendo a propriedade de especificação, temos

$$
\frac{\tau\left(x_{0}^{k-1}\right)}{k} \underset{k \rightarrow \infty}{\stackrel{\text { q.c. }}{\longrightarrow}} 1
$$

para quase toda sequência $x=\left(x_{1}, x_{2}, \cdots\right)$.

A função primeiro retorno possível também foi estudada em ([5]), onde provou-se que, para processos independentes e identicamente distribuídos e para qualquer alfabeto $\mathcal{A}$,

$$
k-\tau\left(x_{0}^{k-1}\right) \Rightarrow \mathcal{L}\left(\mu, \sigma^{2}\right),
$$

ou seja, $k-\tau\left(x_{0}^{k-1}\right)$ converge em distribuição para uma lei não degenerada com média $\mu$ e variância $\sigma^{2}$.

O mesmo resultado foi obtido em ([24]) para processos $\beta$-misturadores, tal que $\sum_{j} \ln (j) \beta(j)<$ $\infty$. Além disso, considerando a lei dos grandes desvios, obtemos por ([19]), ([7]) e ([12]) que

$$
\frac{1}{k} \log \mathbb{P}\left(\left|\frac{\tau\left(x_{0}^{k-1}\right)}{k}-1\right|>\epsilon\right)
$$

converge para uma função da entropia de Renyi. 


\subsubsection{Função Tempo de Entrada}

A função primeiro retorno possível também aparece quando se considera o tempo de entrada, dado em (1.1), que é o tempo decorrido até a primeira ocorrência da palavra $x_{0}^{k-1}$ em uma amostra de tamanho $n$. Para um processo de renovação recorrente positivo e para toda sequência, foi provado em ([13]) que, quando a palavra não se sobrepõe, o tempo de entrada pode ser bem aproximado por uma lei exponencial com parâmetro determinado pela medida da palavra ([23]). Por outro lado, quando a palavra se sobrepõe, o parâmetro da lei exponencial é corrigido para $\lambda\left(x_{0}^{k-1}\right) p\left(x_{0}^{k-1}\right)$, onde $p\left(x_{0}^{k-1}\right)$ é a medida da palavra de tamanho $k$ tal que

$$
p\left(x_{0}^{k-1}\right)=\mathbb{P}\left(X_{0}^{k-1}=x_{0}^{k-1}\right)
$$

e $\lambda\left(x_{0}^{k-1}\right)$ é a probabilidade de saída, como definida em (1.3).

\subsubsection{Função Tempo de retorno}

Em ([13]) também foi provado que, para um processo de renovação recorrente positivo e para toda sequência, considerando uma palavra de tamanho $k$ fixa, $x_{0}^{k-1}$, o tempo de retorno também pode ser bem aproximado por uma lei exponencial cujo parâmetro, quando a palavra não tem sobreposição, é a medida da palavra. No entanto, quando a palavra se sobrepõe, a distribuição do tempo de retorno de $x_{0}^{k-1}$ é aproximadamente uma combinação convexa entre uma medida de Dirac na origem e uma lei exponencial com parâmetro $\lambda\left(x_{0}^{k-1}\right) p\left(x_{0}^{k-1}\right)$. O peso desta combinação convexa é $\lambda\left(x_{0}^{k-1}\right)$. Estes resultados foram obtidos para processos $\alpha$-mixing ([4]) e $\phi$-mixing ([8]).

\subsubsection{Tipicalidade de larga-escala}

Como uma ferramenta para provar consistência, o estudo de tipicalidade de larga-escala, ou seja, a questão de quão perto estão as probabilidades empíricas de blocos de tamanho $k$ definidas por

$$
\hat{p}\left(x_{0}^{k-1}\right)=\frac{N_{n}\left(x_{0}^{k-1}\right)}{n-k+1}
$$

onde

$$
N_{n}\left(x_{0}^{k-1}\right)=\sum_{j=0}^{n-k} \mathbb{1}\left\{X_{j}^{j+k-1}=x_{0}^{k-1}\right\} .
$$

das verdadeiras probabilidades (estacionárias), $p\left(x_{0}^{k-1}\right)$, quando o tamanho da amostra $n$ vai para infinito e $k$ pode crescer quando $n$ cresce, foi iniciado em ([15]) para o caso particular de cadeias de Markov.

Foi mostrado que, para uma cadeia de Markov ergódica de qualquer ordem $k_{0}$, a distribuição empírica do bloco de tamanho $k, \hat{p}\left(x_{0}^{k-1}\right)$ está próxima da verdadeira distribuição, $p\left(x_{0}^{k-1}\right)$, eventualmente quase certamente, quando $n \rightarrow \infty$ e $k<\alpha \log n$, para $\alpha$ adequado. Aqui, "eventualmente quase certamente" significa que, com probabilidade 1, existe um patamar $n_{0}$ (dependente da realização $\omega$ ) tal que a afirmação vale para todo $n \geq n_{0}$.

Dando continuidade em ([21]), para cadeias de Markov de ordem $k_{0}$, com alfabeto finito $\mathcal{A}$, teoremas limites foram provados sobre frequências relativas de blocos de tamanho $k$ e de símbolos precedidos por um dado bloco de tamanho $k$, ou seja,

$$
\hat{\mathbb{P}}_{n}\left(X_{k}=x_{k} \mid X_{0}^{k-1}=x_{0}^{k-1}\right),
$$

onde $x_{k} \in \mathcal{A}$ é o símbolo e $k$ pode crescer quando o tamanho da amostra $n$ cresce. Primeiramente uma melhoria do teorema da tipicalidade forte (apresentado em ([15])) foi provada usando a abordagem de grandes desvios, que também funciona para certos processos não-Markovianos. Em seguida, a questão da tipicalidade condicional de larga escala foi abordada para cadeias de Markov irredutíveis de ordem $k_{0}$, isto é, o problema de quão perto estão as probabilidades condicionais 
empíricas

$$
\hat{\mathbb{P}}_{n}\left(X_{k}=x_{k} \mid X_{0}^{k-1}=x_{0}^{k-1}\right)=\frac{\hat{\mathbb{P}}_{n}\left(X_{0}^{k}=x_{0}^{k}\right)}{\hat{\mathbb{P}}_{n-1}\left(X_{0}^{k-1}=x_{0}^{k-1}\right)}=\frac{N_{n}\left(X_{0}^{k}=x_{0}^{k}\right)}{N_{n-1}\left(X_{0}^{k-1}=x_{0}^{k-1}\right)} .
$$

das verdadeiras probabilidades condicionais $\mathbb{P}\left(X_{k}=x_{k} \mid X_{0}^{k-1}=x_{0}^{k-1}\right)$ quando $k \geq k_{0}$ pode crescer com $n$.

\subsection{Objetivos e Resultados Obtidos}

O objetivo deste trabalho é estudar o comportamento assintótico de $\rho\left(x_{0}^{k-1}\right)$ e de um estimador $\hat{\rho}\left(x_{0}^{k-1}\right)$.

O crescimento linear de $\tau\left(x_{0}^{k-1}\right)$ dado em (1.5) nos leva, por um lado, a introduzir o valor de

$$
\tilde{\rho}\left(x_{0}^{k-1}\right)=\mathbb{P}\left(X_{k}^{2 k-1}=x_{0}^{k-1} \mid X_{0}^{k-1}=x_{0}^{k-1}\right)
$$

e utilizá-lo como uma primeira aproximação.

No Capítulo 2, apresentaremos os resultados assintóticos de $\rho\left(x_{0}^{k-1}\right)$. Para isto é fundamental entender o comportamento de $\tau\left(x_{0}^{k-1}\right)$ e de $p\left(x_{0}^{k-1}\right)$. Então, lembraremos o Teorema de Shannon-Mc Millan Breiman para $p\left(x_{0}^{k-1}\right)$, dado por

Teorema 1.1. Suponha que o processo $\left(X_{m}\right)_{m \in \mathbb{N}}$ é ergódico, estacionário e com entropia positiva. Temos que

$$
-\frac{1}{k} \log \mathbb{P}\left(X_{0}^{k-1}=x_{0}^{k-1}\right) \underset{k \rightarrow \infty}{\text { q.c. }} h,
$$

onde h é a entropia métrica do processo.

Este teorema indica que, se o processo tiver boas condições de mistura, $\tilde{\rho}\left(x_{0}^{k-1}\right)$ deveria se comportar como $p\left(x_{0}^{k-1}\right)$ pois

$$
\tilde{\rho}\left(x_{0}^{k-1}\right)=\mathbb{P}\left(X_{k}^{2 k-1}=x_{0}^{k-1} \mid X_{0}^{k-1}=x_{0}^{k-1}\right) \approx \frac{p\left(x_{0}^{k-1}\right) p\left(x_{0}^{k-1}\right)}{p\left(x_{0}^{k-1}\right)}=p\left(x_{0}^{k-1}\right) .
$$

Além disso, o crescimento linear de $\tau\left(x_{0}^{k-1}\right)$ dado em (1.5) nos leva a acreditar que $\tilde{\rho}\left(x_{0}^{k-1}\right)$ possui um comportamento parecido com $\rho\left(x_{0}^{k-1}\right)$. Desta maneira, esperamos para $\tilde{\rho}\left(x_{0}^{k-1}\right)$ e $\rho\left(x_{0}^{k-1}\right)$ um teorema similar ao Teorema de Shannon-Mc Millan-Breinman.

Dentre os resultados apresentados, mostraremos que, sob determinadas condições, o processo satisfaz

$$
-\frac{1}{k} \log \tilde{\rho}\left(x_{0}^{k-1}\right) \underset{k \rightarrow \infty}{\stackrel{\text { q.c. }}{\longrightarrow}} h .
$$

Resultados similares são obtidos para $\rho\left(x_{0}^{k-1}\right)$. Porém, através da construção de um contraexemplo, mostraremos que (1.6) não é válido em geral para $\tilde{\rho}\left(x_{0}^{k-1}\right)$. Não sabemos se a mesma conclusão é válida para $\rho\left(x_{0}^{k-1}\right)$.

No Capítulo 3, serão apresentados os resultados assintóticos para os estimadores $\hat{\tilde{\rho}}\left(x_{0}^{k-1}\right) \mathrm{e}$ $\hat{\rho}\left(x_{0}^{k-1}\right)$. Para uma realização de $n$ passos de um processo estacionário dependente, vamos propor os seguintes estimadores de máxima verossimilhança

$$
\hat{\tilde{\rho}}_{n}\left(x_{0}^{k-1}\right)=\frac{N_{n}\left(x_{0}^{k-1} x_{0}^{k-1}\right)}{N_{n-k}\left(x_{0}^{k-1}\right)}
$$

$\mathrm{e}$

$$
\hat{\rho}_{n}\left(x_{0}^{k-1}\right)=\frac{N_{n}\left(x_{0}^{k-1} x_{k-\tau\left(x_{0}^{k-1}\right)}^{k-1}\right)}{N_{n-\tau\left(x_{0}^{k-1}\right)}\left(x_{0}^{k-1}\right)}
$$


onde $N_{n}(y)$ é a quantidade de vezes que a sequência $y$ aparece na amostra de tamanho $n$ e $N_{n}(x y)$ é a quantidade de vezes que a sequência $x$, seguida da sequência $y$, aparece na amostra de tamanho $n$.

Utilizando o teorema 2 de ([21]) é possível encontrar, sob determinadas condições, o limite superior para a diferença

$$
\left|\hat{\mathbb{P}}_{n}\left(X_{k}=x_{k} \mid X_{0}^{k-1}=x_{0}^{k-1}\right)-\mathbb{P}_{n}\left(X_{k}=x_{k} \mid X_{k-k_{0}}^{k-1}=x_{k-k_{0}}^{k-1}\right)\right|
$$

onde $x_{k} \in \mathcal{A}$ e $k_{0}$ é a ordem da cadeia de Markov.

Nesta tese, estudaremos as propriedades da probabilidade de uma determinada sequência $x_{0}^{k-1}$ se sobrepor sobre uma cópia dela mesma. Para isto, iremos demonstrar, sob determinadas condições, uma extensão do teorema mencionado acima, porém, aplicado a árvores de contexto e trabalhando com a probabilidade de permanecer em $x_{0}^{k-1}$. Note que em ([21]), o teorema é demonstrado para cadeias de Markov irredutíveis de ordem $k_{0}$, considerando a probabilidade condicional de um único símbolo $x_{k}$ aparecer dado que uma determinada sequência $x_{0}^{k-1}$ apareceu anteriormente. 


\section{Capítulo 2}

\section{Propriedades assintóticas}

Neste capítulo provaremos as propriedades assintóticas de $\tilde{\rho}_{n}\left(x_{0}^{k-1}\right)$ e $\rho_{n}\left(x_{0}^{k-1}\right)$. O ingrediente principal da prova é o Teorema de Shannon-Mc Millan-Breiman ([11]). Na primeira seção deste capítulo, algumas definições e teoremas serão apresentados. A seguir, mostraremos os resultados obtidos e suas respectivas demonstrações. Finalmente, um contra-exemplo será construído, com o intuito de mostrar que estes resultados não são válidos para casos mais gerais.

\subsection{Definições}

Nesta seção, serão apresentadas algumas definições utilizadas no decorrer do capítulo.

Consideraremos um processo estocástico estacionário $\left(X_{m}\right)_{m \in \mathbb{N}}$ tomando valores no alfabeto finito $\mathcal{A}$. O espaço $\mathcal{A}^{\mathbb{N}}$ das realizações deste processo será denotado $\Omega$.

Definição 2.1. Definimos o Tempo de Entrada da palavra $x_{0}^{k-1}$ como sendo a variável aleatória $T_{x_{0}^{k-1}}: \Omega \rightarrow \mathbb{N} \cup\{\infty\}$ dada por:

$$
T_{x_{0}^{k-1}}(\omega)=\inf \left\{j \geq 1: X_{j}^{j+k-1}(\omega)=x_{0}^{k-1}\right\} .
$$

Observe que o tempo de entrada de uma palavra é o menor deslocamento necessário para se encontrar a palavra $x_{0}^{k-1}$ em uma realização específica $\omega$ do processo.

Definição 2.2. Definimos o Primeiro Retorno possivel da palavra $x_{0}^{k-1}$ como

$$
\tau\left(x_{0}^{k-1}\right)=\inf _{\left\{\omega: w_{0}^{k-1}=x_{0}^{k-1}\right\}} T_{x_{0}^{k-1}}(\omega) .
$$

Observe que o primeiro tempo de retorno possível de $x_{0}^{k-1}$ é o ínfimo entre os tempos de entrada de todas as sequências que começam em $x_{0}^{k-1}$.

Exemplo 2.3. Considere $x_{0}^{6}=a a b a a a b$.

$\begin{array}{llllllllllll}\quad \text { Palavra: } & a & a & b & a & \boldsymbol{a} & \boldsymbol{a} & \boldsymbol{b} & & & & \\ 1 \text { deslocamento: } & & a & a & b & a & a & a & b & & & \\ \text { 2 deslocamentos: } & & & a & a & b & a & a & a & b & & \\ \text { 3 deslocamentos: } & & & & a & a & b & a & a & a & b & \\ \text { 4 deslocamentos: } & & & & & \boldsymbol{a} & \boldsymbol{a} & \boldsymbol{b} & a & a & a & b\end{array}$

Observe que, neste exemplo, o encaixe ocorreu após 4 deslocamentos à direita. Logo, $\tau(a a b a a a b)=$ 4.

Podemos definir agora as probabilidades de saída e de permanência em $x_{0}^{k-1}$. 
Definição 2.4. A Probabilidade de Saída de $x_{0}^{k-1}$ é definida como

$$
\lambda\left(x_{0}^{k-1}\right)=\mathbb{P}\left(X_{\tau\left(x_{0}^{k-1}\right)}^{\tau\left(x^{k-1}\right)+k-1} \neq x_{0}^{k-1} \mid X_{0}^{k-1}=x_{0}^{k-1}\right) .
$$

Definição 2.5. A Probabilidade de permanência dentro do conjunto $x_{0}^{k-1}$ é definida como

$$
\rho\left(x_{0}^{k-1}\right)=1-\lambda\left(x_{0}^{k-1}\right)=\mathbb{P}\left(X_{\tau\left(x_{0}^{k-1}\right)}^{\tau\left(x^{k-1}\right)+k-1}=x_{0}^{k-1} \mid X_{0}^{k-1}=x_{0}^{k-1}\right) .
$$

Definição 2.6. Similarmente, definimos

$$
\tilde{\rho}\left(x_{0}^{k-1}\right)=\mathbb{P}\left(X_{k}^{2 k-1}=x_{0}^{k-1} \mid X_{0}^{k-1}=x_{0}^{k-1}\right) .
$$

Com o intuito de facilitar a leitura, na próxima seção apresentaremos alguns conceitos de dependência de variáveis aleatórias utilizados neste trabalho.

\subsubsection{Definições relativas à dependência}

Considere $\left(X_{m}\right)_{m \in \mathbb{N}}$ um processo estocástico estacionário com valores no alfabeto $\mathcal{A}$. O valor de $X_{n}$ é interpretado como o estado do processo no tempo $n$. As palavras serão vistas como realizações deste processo.

Denote $\mathcal{F}_{n}^{m}$ a $\sigma$-álgebra gerada pelas palavras $\left\{X_{n}^{m}=x_{n}^{m}\right\}$. O processo $\left(X_{m}\right)_{m \in \mathbb{N}}$ é

1. $\psi_{g}$-regular se $\exists g \in \mathbb{N}$ tal que, sendo

$$
f_{1}(m, n)=\inf _{A \in \mathcal{F}_{1}^{n}, B \in \mathcal{F}_{n+1+g}^{n+1+g+m}}\left|\frac{\mathbb{P}(B \mid A)}{\mathbb{P}(B)}\right|, \sup _{A \in \mathcal{F}_{1}^{n}, B \in \mathcal{F}_{n+1+g}^{n+1+g+m}}\left|\frac{\mathbb{P}(B \mid A)}{\mathbb{P}(B)}\right|=f_{2}(m, n)
$$

tem-se que $\frac{1}{m} \log f_{1}(m, c m)$ e $\frac{1}{m} \log f_{2}(m, c m)$ convergem para 0 quando $m \rightarrow \infty$, para toda constante positiva $c$.

Os processos $\psi_{g^{-}}$-regulares foram introduzidos em ([12]), onde é mostrado que a condição $\psi_{g^{-}}$ regular implica na condição $\psi_{g+1}$-regular, para qualquer inteiro positivo $g$. Nesse trabalho, é construído um processo que satisfaz a condição $\psi_{1}$-regular mas que não satisfaz a condição $\psi_{0}$-regular, indicando como construir processos que satisfazem a condição $\psi_{g+1}$-regular e que não satisfazem a condição $\psi_{g}$-regular, para qualquer inteiro positivo $g$.

Nos teoremas da seção 2.2, iremos utilizar, em particular, a propriedade $\psi_{g}$-regular, com $g=0$.

2. processo de renovação se, para qualquer estado $x_{n+1} \in \mathcal{A}$ :

$$
\mathbb{P}\left(X_{n+1}=x_{n+1} \mid X_{1}^{n}=0^{n}, X_{0}=1\right)=\mathbb{P}\left(X_{n+1}=x_{n+1} \mid X_{1}^{n}=0^{n}, X_{0}=1, X_{-\infty}^{-1}=x_{-\infty}^{-1}\right) .
$$

\subsubsection{Preliminares}

Nesta seção apresentaremos dois teoremas já conhecidos na literatura, que serão utilizados no decorrer do capítulo. O primeiro é o Teorema de Shannon-Mc Millan-Breiman ([11]) e o segundo teorema foi apresentado em ([29]) e ([9]), que demonstra que $\tau\left(x_{0}^{k-1}\right)$ tem um comportamento próximo de $k$, o tamanho da palavra.

Teorema 2.7. Suponha que o processo $\left(X_{m}\right)_{m \in \mathbb{N}}$ é ergódico, estacionário e com entropia positiva h. Temos que

$$
-\frac{1}{k} \log \mathbb{P}\left(X_{0}^{k-1}=x_{0}^{k-1}\right) \underset{k \rightarrow \infty}{\stackrel{q . c .}{\longrightarrow}} h .
$$


Teorema 2.8. Suponha que $\left(X_{m}\right)_{m \in \mathbb{N}}$ é um processo ergódico com alfabeto finito ou contável e com entropia positiva, que satisfaz a propriedade de especificação. Então, temos que

$$
\frac{\tau\left(x_{0}^{k-1}\right)}{k} \underset{k \rightarrow \infty}{\stackrel{\text { q.c. }}{\longrightarrow}} 1 \text {. }
$$

\subsection{Propriedades assintóticas de $\tilde{\rho}\left(x_{0}^{k-1}\right)$ e $\rho\left(x_{0}^{k-1}\right)$ para processos $\psi_{0}$-regulares.}

O teorema a seguir nos mostra que, para $\left(X_{m}\right)_{m \in \mathbb{N}}$ um processo ergódico, estacionário e que satisfaz a condição $\psi_{0}$-regular, temos que a probabilidade condicional

$$
\tilde{\rho}\left(x_{0}^{k-1}\right)=\mathbb{P}\left(X_{k}^{2 k-1}=x_{0}^{k-1} \mid X_{0}^{k-1}=x_{0}^{k-1}\right)
$$

apresenta decaimento exponencial a medida que o tamanho da palavra cresce, para quase toda sequência e com taxa exponencial dada pela entropia do processo.

Teorema 2.9. Para um processo $\left(X_{m}\right)_{m \in \mathbb{N}}$ ergódico, estacionário e que satisfaz a condição $\psi_{0^{-}}$ regular, temos

$$
-\frac{1}{k} \log \tilde{\rho}\left(x_{0}^{k-1}\right) \underset{k \rightarrow \infty}{\stackrel{q . c .}{\longrightarrow}} h .
$$

Prova. Por definição, temos que

$$
-\frac{1}{k} \log \tilde{\rho}\left(x_{0}^{k-1}\right)=-\frac{1}{k} \log \frac{\mathbb{P}\left(X_{k}^{2 k-1}=x_{0}^{k-1} \mid X_{0}^{k-1}=x_{0}^{k-1}\right)}{\mathbb{P}\left(X_{k}^{2 k-1}=x_{0}^{k-1}\right)}-\frac{1}{k} \log \mathbb{P}\left(X_{k}^{2 k-1}=x_{0}^{k-1}\right) .
$$

Como o processo é estacionário e satisfaz a condição $\psi_{0}$-regular, utilizando o Teorema 2.7, temos que

$$
-\frac{1}{k} \log \tilde{\rho}\left(x_{0}^{k-1}\right) \leq-\frac{1}{k} \log f_{1}(k, k)-\frac{1}{k} \log \mathbb{P}\left(X_{0}^{k-1}=x_{0}^{k-1}\right) \underset{k \rightarrow \infty}{\text { q.c. }} \rightarrow 0+h=h
$$

e, do mesmo modo,

$$
-\frac{1}{k} \log \tilde{\rho}\left(x_{0}^{k-1}\right) \geq-\frac{1}{k} \log f_{2}(k, k)-\frac{1}{k} \log \mathbb{P}\left(X_{0}^{k-1}=x_{0}^{k-1}\right) \underset{k \rightarrow \infty}{\stackrel{\text { q.c. }}{\longrightarrow}} 0+h=h .
$$

De forma semelhante, de acordo com o próximo teorema, temos que para $\left(X_{m}\right)_{m \in \mathbb{N}}$ um processo ergódico, estacionário e que satisfaz a condição $\psi_{0}$-regular, a probabilidade condicional

$$
\rho\left(x_{0}^{k-1}\right)=\mathbb{P}\left(X_{\tau\left(x_{0}^{k-1}\right)}^{\tau\left(x^{k-1}\right)+k-1}=x_{0}^{k-1} \mid X_{0}^{k-1}=x_{0}^{k-1}\right)
$$

apresenta decaimento exponencial a medida que o tamanho da palavra cresce, para quase toda sequência e com taxa exponencial dada pela entropia do processo.

Teorema 2.10. Para um processo $\left(X_{m}\right)_{m \in \mathbb{N}}$ ergódico, estacionário e que satisfaz a condição $\psi_{0^{-}}$ regular, temos

$$
-\frac{1}{k} \log \rho\left(x_{0}^{k-1}\right) \underset{k \rightarrow \infty}{\stackrel{q . c .}{\longrightarrow}} h .
$$

Prova. Por definição, temos que

$\rho\left(x_{0}^{k-1}\right)=\mathbb{P}\left(X_{\tau\left(x_{0}^{k-1}\right)}^{\tau\left(x_{0}^{k-1}\right)+k-1}=x_{0}^{k-1} \mid X_{0}^{k-1}=x_{0}^{k-1}\right)=\frac{\mathbb{P}\left(X_{0}^{\tau\left(x_{0}^{k-1}\right)-1}=x_{0}^{\tau\left(x_{0}^{k-1}\right)-1} \cap X_{\tau\left(x_{0}^{\tau-1}\right)}^{\tau\left(x_{k-1}^{k-1}\right)+k-1}=x_{0}^{k-1}\right)}{\mathbb{P}\left(X_{0}^{k-1}=x_{0}^{k-1}\right)}$ 
Multiplicando e dividindo pelo termo $\mathbb{P}\left(X_{\tau\left(x_{0}^{k-1}\right)}^{\tau\left(x^{k-1}\right)+k-1}=x_{0}^{k-1}\right)$, podemos reescrever a expressão acima da seguinte forma

$$
\frac{\mathbb{P}\left(X_{\tau\left(x_{0}^{k-1}\right)}^{\tau\left(x_{k-1}^{k-1}\right)+k-1}=x_{0}^{k-1} \mid X_{0}^{\tau\left(x_{0}^{k-1}\right)-1}=x_{0}^{\tau\left(x_{0}^{k-1}\right)-1}\right)}{\mathbb{P}\left(X_{\tau\left(x_{0}^{k-1}\right)}^{\tau\left(x_{0}^{k-1}\right)+k-1}=x_{0}^{k-1}\right)} \mathbb{P}\left(X_{0}^{\tau\left(x_{0}^{k-1}\right)-1}=x_{0}^{\tau\left(x_{0}^{k-1}\right)-1}\right) \frac{\mathbb{P}\left(X_{\tau\left(x_{0}^{k-1}\right)}^{\tau\left(x_{k-1}^{k-1}\right)+k-1}=x_{0}^{k-1}\right)}{\mathbb{P}\left(X_{0}^{k-1}=x_{0}^{k-1}\right)} .
$$

Logo, como o processo é estacionário e satisfaz a condição $\psi_{0}$-regular, utilizando os teoremas 2.7 e 2.8 , temos que

$$
\begin{aligned}
-\frac{1}{k} \log \rho\left(x_{0}^{k-1}\right) & \leq-\frac{1}{k} \log f_{1}\left(\tau\left(x_{0}^{k-1}\right), k\right)-\frac{1}{k} \log \mathbb{P}\left(X_{0}^{\tau\left(x_{0}^{k-1}\right)-1}=x_{0}^{\tau\left(x_{0}^{k-1}\right)-1}\right) \\
& -\frac{1}{k} \log \mathbb{P}\left(X_{0}^{k-1}=x_{0}^{k-1}\right)+\frac{1}{k} \log \mathbb{P}\left(X_{0}^{k-1}=x_{0}^{k-1}\right)=-\frac{1}{k} \log f_{1}\left(\tau\left(x_{0}^{k-1}\right), k\right) \\
& -\frac{\tau\left(x_{0}^{k-1}\right)}{k} \frac{1}{\tau\left(x_{0}^{k-1}\right)} \log \mathbb{P}\left(X_{0}^{\tau\left(x_{0}^{k-1}\right)-1}=x_{0}^{\tau\left(x_{0}^{k-1}\right)-1}\right) \underset{k \rightarrow \infty}{\text { q.c. }} \rightarrow+1 h=h
\end{aligned}
$$

e, do mesmo modo,

$$
\begin{aligned}
-\frac{1}{k} \log \rho\left(x_{0}^{k-1}\right) & \geq-\frac{1}{k} \log f_{2}\left(\tau\left(x_{0}^{k-1}\right), k\right)-\frac{1}{k} \log \mathbb{P}\left(X_{0}^{\tau\left(x_{0}^{k-1}\right)-1}=x_{0}^{\tau\left(x_{0}^{k-1}\right)-1}\right) \\
& -\frac{1}{k} \log \mathbb{P}\left(X_{0}^{k-1}=x_{0}^{k-1}\right)+\frac{1}{k} \log \mathbb{P}\left(X_{0}^{k-1}=x_{0}^{k-1}\right)=-\frac{1}{k} \log f_{2}\left(\tau\left(x_{0}^{k-1}\right), k\right) \\
& -\frac{\tau\left(x_{0}^{k-1}\right)}{k} \frac{1}{\tau\left(x_{0}^{k-1}\right)} \log \mathbb{P}\left(X_{0}^{\tau\left(x_{0}^{k-1}\right)-1}=x_{0}^{\tau\left(x_{0}^{k-1}\right)-1}\right) \underset{k \rightarrow \infty}{\text { q.c. }} \longrightarrow+1 h=h .
\end{aligned}
$$

\subsection{Propriedades assintóticas de $\tilde{\rho}\left(x_{0}^{k-1}\right)$ e $\rho\left(x_{0}^{k-1}\right)$ para processos $\psi_{g}$-regulares, com $g>0$.}

De acordo com o próximo teorema temos que, para $\left(X_{m}\right)_{m \in \mathbb{N}}$ um processo ergódico, estacionário e que satisfaz a condição $\psi_{g}$-regular, então $-\frac{1}{k} \log \tilde{\rho}\left(x_{0}^{k-1}\right)$ é limitada inferiormente pela função

$$
-\frac{1}{k} \log f_{2}(k, k-g)-\frac{1}{k} \log \mathbb{P}\left(X_{0}^{k-g-1}=x_{0}^{k-g-1}\right)
$$

que apresenta decaimento exponencial a medida que o tamanho da palavra cresce, para quase toda sequência e com taxa exponencial dada pela entropia do processo.

Teorema 2.11. Para um processo $\left(X_{m}\right)_{m \in \mathbb{N}}$ ergódico, estacionário e que satisfaz a condição $\psi_{g^{-}}$ regular, temos

$$
\lim _{k \rightarrow \infty}-\frac{1}{k} \log \tilde{\rho}\left(x_{0}^{k-1}\right) \geq h, \quad \text { q.c. }
$$

Prova. Por definição, temos que

$$
\tilde{\rho}\left(x_{0}^{k-1}\right)=\frac{\mathbb{P}\left(X_{0}^{k-1}=x_{0}^{k-1} \cap X_{k}^{2 k-1}=x_{0}^{k-1}\right)}{\mathbb{P}\left(X_{0}^{k-1}=x_{0}^{k-1}\right)} .
$$


PROPRIEDADES ASSINTÓTICAS DE $\tilde{\rho}\left(X_{0}^{K-1}\right)$ E $\rho\left(X_{0}^{K-1}\right)$ PARA PROCESSOS $\psi_{G}$-REGULARES, COM

Para $0 \leq g<k$, vale

$$
\mathbb{P}\left(X_{0}^{k-1}=x_{0}^{k-1} \cap X_{k}^{2 k-1}=x_{0}^{k-1}\right) \leq \mathbb{P}\left(X_{0}^{k-g-1}=x_{0}^{k-g-1} \cap X_{k}^{2 k-1}=x_{0}^{k-1}\right) .
$$

Portanto, como o processo é estacionário, então

$$
\begin{aligned}
\tilde{\rho}\left(x_{0}^{k-1}\right) & \leq \frac{\mathbb{P}\left(X_{0}^{k-g-1}=x_{0}^{k-g-1} \cap X_{k}^{2 k-1}=x_{0}^{k-1}\right)}{\mathbb{P}\left(X_{0}^{k-1}=x_{0}^{k-1}\right)} \\
& =\frac{\mathbb{P}\left(X_{k}^{2 k-1}=x_{0}^{k-1} \mid X_{0}^{k-g-1}=x_{0}^{k-g-1}\right)}{\mathbb{P}\left(X_{k}^{2 k-1}=x_{0}^{k-1}\right)} \mathbb{P}\left(X_{0}^{k-g-1}=x_{0}^{k-g-1}\right) .
\end{aligned}
$$

Como o processo satisfaz a condição $\psi_{g}$-regular, utilizando o Teorema 2.7, temos que

$-\frac{1}{k} \log \tilde{\rho}\left(x_{0}^{k-1}\right) \geq-\frac{1}{k} \log f_{2}(k, k-g)-\frac{(k-g)}{k} \frac{1}{(k-g)} \log \mathbb{P}\left(X_{0}^{k-g-1}=x_{0}^{k-g-1}\right) \underset{k \rightarrow \infty}{\mathrm{q} . c .} 0+1 h=h$.

De forma parecida ao teorema anterior, temos que para $\left(X_{m}\right)_{m \in \mathbb{N}}$ um processo ergódico, estacionário e que satisfaz a condição $\psi_{g}$-regular, temos que $-\frac{1}{k} \log \rho\left(x_{0}^{k-1}\right)$ é limitada inferiormente pela função

$$
\begin{aligned}
& -\frac{1}{k} \log f_{2}\left(\tau\left(x_{0}^{k-1}\right), k-g\right)-\frac{1}{k} \log \mathbb{P}\left(X_{0}^{\tau\left(x_{0}^{k-1}\right)-1}=x_{0}^{\tau\left(x_{0}^{k-1}\right)-1}\right) \\
& -\frac{1}{k} \log \mathbb{P}\left(X_{0}^{k-g-1}=x_{0}^{k-g-1}\right)+\frac{1}{k} \log \mathbb{P}\left(X_{0}^{k-1}=x_{0}^{k-1}\right)
\end{aligned}
$$

que apresenta decaimento exponencial a medida que o tamanho da palavra cresce, para quase toda sequência e com taxa exponencial dada pela entropia do processo.

Teorema 2.12. Para um processo $\left(X_{m}\right)_{m \in \mathbb{N}}$ ergódico, estacionário e que satisfaz a condição $\psi_{g^{-}}$ regular, temos

$$
\lim _{k \rightarrow \infty}-\frac{1}{k} \log \rho\left(x_{0}^{k-1}\right) \geq h, \quad \text { q.c. }
$$

Prova. Por definição, temos que

$$
\rho\left(x_{0}^{k-1}\right)=\frac{\mathbb{P}\left(X_{0}^{k-1}=x_{0}^{k-1} \cap X_{k}^{k+\tau\left(x_{0}^{k-1}\right)-1}=x_{0}^{\tau\left(x_{0}^{k-1}\right)-1}\right)}{\mathbb{P}\left(X_{0}^{k-1}=x_{0}^{k-1}\right)} .
$$

Para $0 \leq g<k$, vale

$\mathbb{P}\left(X_{0}^{k-1}=x_{0}^{k-1} \cap X_{k}^{k+\tau\left(x_{0}^{k-1}\right)-1}=x_{0}^{\tau\left(x_{0}^{k-1}\right)-1}\right) \leq \mathbb{P}\left(X_{0}^{k-g-1}=x_{0}^{k-g-1} \cap X_{k}^{k+\tau\left(x_{0}^{k-1}\right)-1}=x_{0}^{\tau\left(x_{0}^{k-1}\right)-1}\right)$.

Desta forma, temos que

$$
\begin{aligned}
\rho\left(x_{0}^{k-1}\right) \leq & \frac{\mathbb{P}\left(X_{0}^{k-g-1}=x_{0}^{k-g-1} \cap X_{k}^{k+\tau\left(x_{0}^{k-1}\right)-1}=x_{0}^{\tau\left(x_{0}^{k-1}\right)-1}\right)}{\mathbb{P}\left(X_{0}^{k-1}=x_{0}^{k-1}\right)} \\
& =\frac{\mathbb{P}\left(X_{k}^{k+\tau\left(x_{0}^{k-1}\right)-1}=x_{0}^{\tau\left(x_{0}^{k-1}\right)-1} \mid X_{0}^{k-g-1}=x_{0}^{k-g-1}\right)}{\mathbb{P}\left(X_{0}^{k-1}=x_{0}^{k-1}\right)} \times \mathbb{P}\left(X_{0}^{k-g-1}=x_{0}^{k-g-1}\right) .
\end{aligned}
$$


Multiplicando e dividindo pelo termo $\mathbb{P}\left(X_{k}^{k+\tau\left(x_{0}^{k-1}\right)-1}=x_{0}^{\tau\left(x_{0}^{k-1}\right)-1}\right)$, podemos reescrever a expressão acima da seguinte forma

$$
\frac{\mathbb{P}\left(X_{k}^{k+\tau\left(x_{0}^{k-1}\right)-1}=x_{0}^{\tau\left(x_{0}^{k-1}\right)-1} \mid X_{0}^{k-g-1}=x_{0}^{k-g-1}\right)}{\mathbb{P}\left(X_{k}^{k+\tau\left(x_{0}^{k-1}\right)-1}=x_{0}^{\tau\left(x_{0}^{k-1}\right)-1}\right)} \mathbb{P}\left(X_{0}^{k-g-1}=x_{0}^{k-g-1}\right) \frac{\mathbb{P}\left(X_{k}^{k+\tau\left(x_{0}^{k-1}\right)-1}=x_{0}^{\tau\left(x_{0}^{k-1}\right)-1}\right)}{\mathbb{P}\left(X_{0}^{k-1}=x_{0}^{k-1}\right)} .
$$

Logo, como o processo é estacionário e satisfaz a condição $\psi_{g}$-regular, utilizando os teoremas 2.7 e 2.8 , temos que

$$
\begin{aligned}
-\frac{1}{k} \log \rho\left(x_{0}^{k-1}\right) & \geq-\frac{1}{k} \log f_{2}\left(\tau\left(x_{0}^{k-1}\right), k-g\right)-\frac{\tau\left(x_{0}^{k-1}\right)}{k} \frac{1}{\tau\left(x_{0}^{k-1}\right)} \log \mathbb{P}\left(X_{0}^{\tau\left(x_{0}^{k-1}\right)-1}=x_{0}^{\tau\left(x_{0}^{k-1}\right)-1}\right) \\
& -\frac{(k-g)}{k} \frac{1}{(k-g)} \log \mathbb{P}\left(X_{0}^{k-g-1}=x_{0}^{k-g-1}\right) \\
& +\frac{1}{k} \log \mathbb{P}\left(X_{0}^{k-1}=x_{0}^{k-1}\right) \underset{k \rightarrow \infty}{\stackrel{\text { q.c. }}{\longrightarrow}} 0+1 h+1 h-h=h .
\end{aligned}
$$

A seguir, mostraremos que os teoremas 2.9 e 2.11 acima não são válidos para casos mais gerais.

\subsection{Contra-exemplo: Processo ergódico e de entropia positiva}

Com o intuito de mostrar que os teoremas 2.9 e 2.11 não podem ser estendidos para o caso onde o processo é apenas ergódico, estacionário e de entropia positiva, construímos o seguinte contraexemplo:

Definimos $X_{2 k}$ variáveis aleatórias independentes com distribuição Bernoulli( $\left.p\right)$ e variáveis $Y_{2 k}$ e $Z_{2 k}$ tais que $X_{2 k}=Y_{2 k}=Z_{2 k}$. Considere que

$$
Y_{2 k+1}=Y_{2 k}
$$

e que

$$
Z_{2 k-1}=Z_{2 k}
$$

valem para todo $k$.

Observe que as variáveis são independentes nas coordenadas pares.

Sejam $\mathbb{P}^{\prime}$ e $\mathbb{P}^{\prime \prime}$ as medidas dos processos $Y$ e $Z$, respectivamente. Considere os seguintes conjuntos: $k\}$.

$C_{k}^{\prime}=\left\{x_{j+1}=x_{j}\right.$, para todo $j$ par e $\left.0 \leq j \leq k\right\}$ e $C_{k}^{\prime \prime}=\left\{x_{j-1}=x_{j}\right.$, para todo $j$ par e $0 \leq j \leq$

A seguir, mostraremos que:

(a) As medidas $\mathbb{P}^{\prime}$ e $\mathbb{P}^{\prime \prime}$ não são estacionárias, mas a medida $\mathbb{P}=\frac{\left(\mathbb{P}^{\prime}+\mathbb{P}^{\prime \prime}\right)}{2}$ é estacionária.

Note que:

(i) Se a sequência $x_{0}^{k-1} \in C_{k}^{\prime}$, então

$$
\mathbb{P}^{\prime}\left(X_{0}^{k-1}=x_{0}^{k-1}\right)=\prod_{i=0}^{k / 2} \mathbb{P}\left(X_{2 i}=x_{2 i}\right)= \begin{cases}\mathbb{P}^{\prime}\left(X_{j}^{j+k-1}=x_{0}^{k-1}\right), & \text { se } j \text { par } \\ \mathbb{P}^{\prime \prime}\left(X_{j}^{j+k-1}=x_{0}^{k-1}\right), & \text { se } j \text { ímpar }\end{cases}
$$


e

$$
\mathbb{P}^{\prime \prime}\left(X_{0}^{k-1}=x_{0}^{k-1}\right)=0= \begin{cases}\mathbb{P}^{\prime \prime}\left(X_{j}^{j+k-1}=x_{0}^{k-1}\right), & \text { se } j \text { par } \\ \mathbb{P}^{\prime}\left(X_{j}^{j+k-1}=x_{0}^{k-1}\right), & \text { se } j \text { ímpar }\end{cases}
$$

(ii) Se a sequência $x_{0}^{k-1} \in C_{k}^{\prime \prime}$, temos

$$
\mathbb{P}^{\prime}\left(X_{0}^{k-1}=x_{0}^{k-1}\right)=0= \begin{cases}\mathbb{P}^{\prime}\left(X_{j}^{j+k-1}=x_{0}^{k-1}\right), & \text { se } j \text { par } \\ \mathbb{P}^{\prime \prime}\left(X_{j}^{j+k-1}=x_{0}^{k-1}\right), & \text { se } j \text { ímpar }\end{cases}
$$

e

$$
\mathbb{P}^{\prime \prime}\left(X_{0}^{k-1}=x_{0}^{k-1}\right)=\prod_{i=0}^{k / 2} \mathbb{P}\left(X_{2 i}=x_{2 i}\right)= \begin{cases}\mathbb{P}^{\prime \prime}\left(X_{j}^{j+k-1}=x_{0}^{k-1}\right), & \text { se } j \text { par } \\ \mathbb{P}^{\prime}\left(X_{j}^{j+k-1}=x_{0}^{k-1}\right), & \text { se } j \text { ímpar }\end{cases}
$$

Generalizando, obtemos

$$
\mathbb{P}^{\prime}\left(X_{j}^{j+k-1}=x_{0}^{k-1}\right)= \begin{cases}\mathbb{P}^{\prime}\left(X_{0}^{k-1}=x_{0}^{k-1}\right), & \text { para } j \text { par } \\ \mathbb{P}^{\prime \prime}\left(X_{0}^{k-1}=x_{0}^{k-1}\right), & \text { para } j \text { ímpar }\end{cases}
$$

e

$$
\mathbb{P}^{\prime \prime}\left(X_{j}^{j+k-1}=x_{0}^{k-1}\right)= \begin{cases}\mathbb{P}^{\prime \prime}\left(X_{0}^{k-1}=x_{0}^{k-1}\right), & \text { para } j \text { par } \\ \mathbb{P}^{\prime}\left(X_{0}^{k-1}=x_{0}^{k-1}\right), & \text { para } j \text { ímpar }\end{cases}
$$

(b) Para todo $0<p<1$, o processo $\frac{Y+Z}{2}$ é ergódico e tem entropia positiva.

Considere os seguintes casos:

(i) Para toda sequência $x_{0}^{k-1}$ com pelo menos dois símbolos 0 e dois símbolos 1 que pertence ao conjunto $C_{k}^{\prime}$, temos

$$
\mathbb{P}^{\prime}\left(X_{0}^{k-1}=x_{0}^{k-1}\right) \cong p^{\frac{\# 1^{\prime} s}{2}}(1-p)^{\frac{\# 0^{\prime} s}{2}}
$$

e

$$
\mathbb{P}^{\prime \prime}\left(X_{0}^{k-1}=x_{0}^{k-1}\right)=0
$$

(ii) Para toda a sequência $x_{0}^{k-1}$ com pelo menos dois símbolos 0 e dois símbolos 1 que pertence ao conjunto $C_{k}^{\prime \prime}$, temos

$$
\mathbb{P}^{\prime}\left(X_{0}^{k-1}=x_{0}^{k-1}\right)=0
$$

e

$$
\mathbb{P}^{\prime \prime}\left(X_{0}^{k-1}=x_{0}^{k-1}\right) \cong p^{\frac{\# 1^{\prime} s}{2}}(1-p)^{\frac{\# 0^{\prime} s}{2}}
$$

Para (i) e (ii),

$$
h \cong-\frac{p}{2} \log p-\frac{(1-p)}{2} \log (1-p)+\frac{1}{k} \log 2
$$

(iii) Para a sequência $x_{0}^{k-1}$ formada apenas pelo símbolo 1 , temos

$$
\mathbb{P}^{\prime}\left(X_{j}^{j+k-1}=x_{0}^{k-1}\right)=\mathbb{P}^{\prime \prime}\left(X_{j}^{j+k-1}=x_{0}^{k-1}\right) \cong p^{\frac{\# 1^{\prime} s}{2}} \text {, para todo } j .
$$

Então,

$$
h \cong-\frac{1}{k} \log p^{\frac{k}{2} p}=-\frac{p}{2} \log p .
$$


(iv) Para a sequência $x_{0}^{k-1}$ formada apenas pelo símbolo 0 , temos

$$
\mathbb{P}^{\prime}\left(X_{j}^{j+k-1}=x_{0}^{k-1}\right)=\mathbb{P}^{\prime \prime}\left(X_{j}^{j+k-1}=x_{0}^{k-1}\right) \cong p^{\frac{\# 0^{\prime} s}{2}}, \text { para todo } j .
$$

Assim,

$$
h \cong-\frac{1}{k} \log (1-p)^{\frac{k}{2}(1-p)}=-\frac{(1-p)}{2} \log (1-p) .
$$

Logo, para (i), (ii), (iii) e (iv), temos que $h>0$ para $0<p<1$ e $h=0$ para $p=0$ ou $p=1$.

(c) O processo $\frac{Y+Z}{2}$ não satisfaz a condição $\psi_{g}$-regular, para todo $g \in \mathbb{N}$.

Considere os seguintes casos:

(i) Seja $k$ par e o caso particular onde $x^{(1)}=\left\{x_{0}=0, x_{1}=0, x_{2}=1, x_{3}=1\right\}$ e $x^{(2)}=\left\{x_{k}=\right.$ $\left.0, x_{k+1}=1, x_{k+2}=1, x_{k+3}=0\right\}$.

Então, $\mathbb{P}\left(x^{(1)}\right)=\frac{p(1-p)+0}{2}$ e $\mathbb{P}\left(x^{(2)}\right)=\frac{0+p(1-p)^{2}}{2}$.

Note que

$$
x^{(1)} x^{(2)}=\left\{x_{0}=0, x_{1}=0, x_{2}=1, x_{3}=1, \ldots, x_{k}=0, x_{k+1}=1, x_{k+2}=1, x_{k+3}=0\right\} .
$$

Como $k$ é par, temos que:

- $\mathbb{P}^{\prime}\left(x^{(1)} x^{(2)}\right)=0$ pois, se $x_{k}=0$, então $x_{k+1} \neq 1$;

- $\mathbb{P}^{\prime \prime}\left(x^{(1)} x^{(2)}\right)=0$ pois, se $x_{2}=1$, então $x_{1} \neq 0$.

Logo, $\mathbb{P}\left(x^{(1)} x^{(2)}\right)=0$. Neste caso, para todo $k$ par,

$$
\frac{\mathbb{P}\left(x^{(2)} \mid x^{(1)}\right)}{\mathbb{P}\left(x^{(2)}\right)}=\frac{\mathbb{P}\left(x^{(1)} x^{(2)}\right)}{\mathbb{P}\left(x^{(1)}\right) \mathbb{P}\left(x^{(2)}\right)}=0 .
$$

Pela definição de $\psi_{g}$-regular, temos que $\frac{\mathbb{P}\left(x^{(2)} \mid x^{(1)}\right)}{\mathbb{P}\left(x^{(2)}\right)}$ encontra-se entre duas funções $f_{1}(m, n)$ e $f_{2}(m, n)$ tal que $\frac{1}{m} f_{i}(m, c m) \rightarrow 0$, quando $m \rightarrow \infty$, para $i=1,2$. Porém, para estas sequências $x^{(1)}$ e $x^{(2)}$, com $k$ par, isto não é válido pois $\frac{\mathbb{P}\left(x^{(2)} \mid x^{(1)}\right)}{\mathbb{P}\left(x^{(2)}\right)}=0$ e

$$
\text { não existe } \lim _{m \rightarrow \infty} \frac{1}{m} \log \frac{\mathbb{P}\left(x^{(2)} \mid x^{(1)}\right)}{\mathbb{P}\left(x^{(2)}\right)} \text {. }
$$

(ii) Seja $k$ ímpar e o caso particular onde $x^{(1)}=\left\{x_{0}=0, x_{1}=0, x_{2}=1, x_{3}=1\right\}$ e $x^{(2)}=\left\{x_{k}=1, x_{k+1}=1, x_{k+2}=0, x_{k+3}=0\right\}$.

Então, $\mathbb{P}\left(x^{(1)}\right)=\frac{p(1-p)+0}{2}$ e $\mathbb{P}\left(x^{(2)}\right)=\frac{p(1-p)+0}{2}$.

Note que

$$
x^{(1)} x^{(2)}=\left\{x_{0}=0, x_{1}=0, x_{2}=1, x_{3}=1, \ldots, x_{k}=1, x_{k+1}=1, x_{k+2}=0, x_{k+3}=0\right\} .
$$


Como $k$ é ímpar, temos que:

- $\mathbb{P}^{\prime}\left(x^{(1)} x^{(2)}\right)=0$ pois, se $x_{k+1}=1$, então $x_{k+2} \neq 0$;

- $\mathbb{P}^{\prime \prime}\left(x^{(1)} x^{(2)}\right)=0$ pois, se $x_{2}=1$, então $x_{1} \neq 0$.

Logo, $\mathbb{P}\left(x^{(1)} x^{(2)}\right)=0$. Neste caso, para todo $k$ ímpar,

$$
\frac{\mathbb{P}\left(x^{(2)} \mid x^{(1)}\right)}{\mathbb{P}\left(x^{(2)}\right)}=\frac{\mathbb{P}\left(x^{(1)} x^{(2)}\right)}{\mathbb{P}\left(x^{(1)}\right) \mathbb{P}\left(x^{(2)}\right)}=0 .
$$

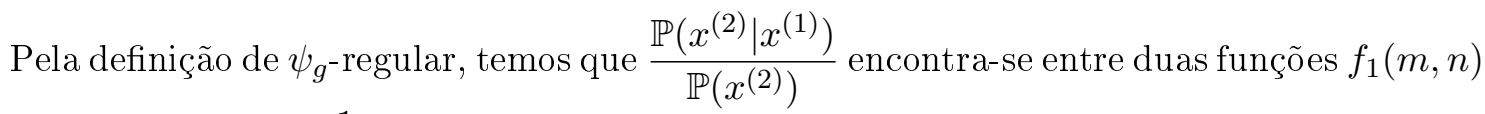
e $f_{2}(m, n)$ tal que $\frac{1}{m} f_{i}(m, c m) \rightarrow 0$, quando $m \rightarrow \infty$, para $i=1,2$. Porém, para estas sequências $x^{(1)}$ e $x^{(2)}$, com $k$ ímpar, isto não é válido pois $\frac{\mathbb{P}\left(x^{(2)} \mid x^{(1)}\right)}{\mathbb{P}\left(x^{(2)}\right)}=0 \mathrm{e}$

$$
\text { não existe } \lim _{m \rightarrow \infty} \frac{1}{m} \log \frac{\mathbb{P}\left(x^{(2)} \mid x^{(1)}\right)}{\mathbb{P}\left(x^{(2)}\right)} \text {. }
$$

(d) O processo $\frac{Y+Z}{2}$ não satisfaz a condição

$$
-\frac{1}{k} \log \tilde{\rho}\left(w_{k}\right) \rightarrow h \quad \text { q.c., } \quad \text { quando } k \rightarrow \infty
$$

onde $h$ é a entropia do processo.

Como prova, iremos mostrar que existem infinitas subsequências $w_{k_{i}(\omega)}$ tal que $p\left(w_{k_{i}(\omega)}\right)>0$ e $p\left(w_{k_{i}(\omega)} w_{k_{i}(\omega)}\right)=0$.

Seja $w_{k}$ uma sequência finita contida em $\mathbb{M}$ onde

$\mathbb{M}=\left\{\omega \in \mathcal{A}^{\mathbb{N}}\right.$ tal que $\omega$ começa com 10 e $\omega$ tem infinitos 0 's $\}$.

Neste caso, para $\omega$, existe uma subsequência infinita $k_{1}(\omega), k_{2}(\omega), k_{3}(\omega), \cdots$ onde $w_{k_{i}(\omega)}=0$.

Então,

$$
w_{k_{i}(\omega)}=\underbrace{10 \ldots 0}_{k_{i}(\omega)}
$$

e

$$
w_{k_{i}(\omega)} w_{k_{i}(\omega)}=\underbrace{10 \ldots 010 \ldots 0}_{2 k_{i}(\omega)}
$$

Nestes casos, $p\left(w_{k_{i}(\omega)}\right)>0$ e $p\left(w_{k_{i}(\omega)} w_{k_{i}(\omega)}\right)=0$.

Logo, existem infinitos $k_{i}(\omega)$ tal que $p\left(w_{k_{i}(\omega)}\right)>0$ e $p\left(w_{k_{i}(\omega)} w_{k_{i}(\omega)}\right)=0$, ou seja,

$$
\tilde{\rho}\left(w_{k_{i}(\omega)}\right)=0 .
$$

Consequentemente,

$$
-\frac{1}{k} \log \tilde{\rho}\left(w_{k}\right) \nrightarrow h \quad \text { q.c., quando } k \rightarrow \infty .
$$


Portanto, através deste contra-exemplo, mostramos que existe um processo estacionário, com entropia positiva e que não satisfaz a condição $\psi_{g}$-regular, para todo $g \in \mathbb{N}$ e tal que

$$
-\frac{1}{k} \log \tilde{\rho}\left(w_{k}\right) \nrightarrow h \quad \text { q.c. quando } k \rightarrow \infty .
$$




\section{Capítulo 3}

\section{Propriedades dos Estimadores}

Neste capítulo construiremos e apresentaremos as propriedades dos estimadores $\hat{\tilde{\rho}}_{n}\left(x_{0}^{k-1}\right)$ e $\hat{\rho}_{n}\left(x_{0}^{k-1}\right)$ para árvores de contexto. Inicialmente, alguns conceitos básicos e a estrutura fundamental dos processos utilizados serão apresentados e, nas seções seguintes, provaremos que os estimadores $\hat{\tilde{\rho}}_{n}\left(x_{0}^{k-1}\right)$ e $\hat{\rho}_{n}\left(x_{0}^{k-1}\right)$ convergem para $\tilde{\rho}_{n}\left(x_{0}^{k-1}\right)$ e $\rho_{n}\left(x_{0}^{k-1}\right)$ q.c., respectivamente, quando $n \rightarrow \infty$, onde $n$ é o tamanho da amostra.

\section{1 Árvores de Contexto}

Seja $\left(X_{m}\right)_{m \in \mathbb{N}}$ um processo estocástico, ergódico e estacionário sobre o alfabeto finito $\mathcal{A}$, de tamanho $|\mathcal{A}|$.

Dados dois inteiros $m \leq n$ denotamos por $x_{m}^{n}$ a sequência de símbolos $x_{m}, \cdots, x_{n}$ de $\mathcal{A}$. O comprimento da sequência $x_{m}^{n}$ é definido por $l\left(x_{m}^{n}\right)=n-m+1$. Qualquer sequência $x_{m}^{n}$ com $m>n$ representa a sequência vazia.

Dadas duas sequências $x_{m}^{n}$ e $x_{r}^{s}$, com $l\left(x_{m}^{n}\right)<\infty$, denotamos por $x_{m}^{n} x_{r}^{s}$ a sequência $x_{m}, \cdots, x_{n}, x_{r}, \cdots, x_{s}$, de comprimento $l\left(x_{m}^{n}\right)+l\left(x_{r}^{s}\right)$, obtida pela concatenação das duas sequências.

Definição 3.1. Dizemos que a sequência s é um sufixo da sequência w se existe uma sequência u, com $l(u) \geq 1$, tal que $w=$ us. Neste caso, escrevemos $s \prec w$. Quando $s \prec w$ ou $s=w$, escrevemos $s \preceq w$.

O sufixo de uma sequência semi-infinita é definido similarmente.

Definição 3.2. Uma sequência $w \in \mathcal{A}^{k}$ é um contexto para o processo $\left(X_{m}\right)_{m \in \mathbb{N}}$ se $\mathbb{P}\left(X_{0}^{k-1}=\right.$ $w)>0$ e para toda sequência semi-infinita $x_{-\infty}^{k-1} \in \mathcal{A}^{\mathbb{N}}$ tal que $w$ é um sufixo de $x_{-\infty}^{k-1}$ temos que

$$
\mathbb{P}\left(X_{k}=a \mid X_{-\infty}^{k-1}=x_{-\infty}^{k-1}\right)=\mathbb{P}\left(X_{k}=a \mid X_{0}^{k-1}=w\right)
$$

para todo a $\in \mathcal{A}$ e nenhum sufixo de w satisfaz esta equação.

A definição acima foi extraída de ([16]) e implica que o conjunto de todos os contextos pode ser representado como uma árvore com raiz e rótulos. Esta árvore é chamada árvore de contextos do processo $\left(X_{m}\right)_{m \in \mathbb{N}}$ e será denotada por $\mathcal{T}$.

Definição 3.3. Considere $\mathcal{A}^{j}$ o conjunto de todas as sequências de comprimento $j$ sobre $\mathcal{A}$. Seja

$$
\mathcal{A}^{*}=\bigcup_{j=1}^{\infty} \mathcal{A}^{j} .
$$

Um subconjunto $\mathcal{T}$ de $\mathcal{A}^{*}$ é uma árvore se nenhuma sequência $s \in \mathcal{T}$ é um sufixo de outra sequência $w \in \mathcal{T}$. Esta propriedade é chamada de propriedade do sufixo. 
Denotamos por $d(\mathcal{T})$ a profundidade da árvore $\mathcal{T}$, ou seja,

$$
d(\mathcal{T})=\sup \{l(w): w \in \mathcal{T}\}
$$

No caso onde $d(\mathcal{T})<\infty$, temos que $\mathcal{T}$ tem um número finito de sequências e dizemos que $\mathcal{T}$ é limitada, denotando por $|\mathcal{T}|$ o número de sequências em $\mathcal{T}$. Por outro lado, se $d(\mathcal{T})=\infty$, então $\mathcal{T}$ tem um número contável de sequências e dizemos que $\mathcal{T}$ é ilimitada.

Definição 3.4. A árvore de contexto é irredutivel se nenhuma sequência $w$ pode ser substituída por um sufixo s sem violar a propriedade de sufixo. Esta noção foi introduzida em ([16]) e generaliza o conceito de árvore completa.

Definição 3.5. Uma árvore de contexto probabilistica sobre $\mathcal{A}$ é um par ordenado $(\mathcal{T}, p)$ tal que

(a) $\mathcal{T}$ é uma árvore irredutível.

(b) $p=\{p(. \mid w): w \in \mathcal{T}\}$ é uma família de probabilidades de transição sobre $\mathcal{A}$.

Definição 3.6. Dizemos que o processo $\left(X_{m}\right)_{m \in \mathbb{N}}$ é compativel com a árvore de contexto probabilistica $(\mathcal{T}, \bar{p})$ se e somente se

(a) Todo $w \in \mathcal{T}$ é um contexto para o processo $\left(X_{m}\right)_{m \in \mathbb{N}}$.

(b) Para todo $w \in \mathcal{T}$ e todo $a \in \mathcal{A}, \bar{p}(a \mid w)=\mathbb{P}\left(X_{k}=a \mid X_{k-l(w)}^{k-1}=w\right)$.

A seguir, são apresentadas duas árvores de contexto. A figura 3.1 representa a árvore de contexto limitada, de um processo de renovação com profundidade igual a 3. Já a figura 3.2 representa a árvore de contexto ilimitada de um processo de renovação.

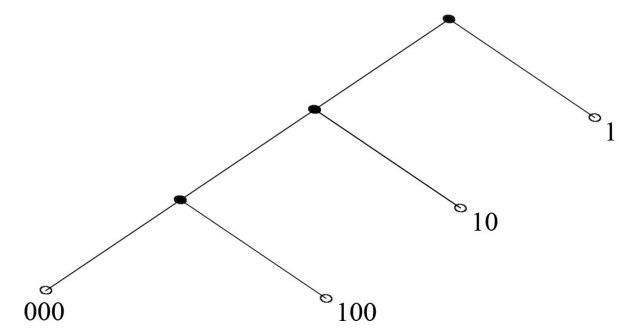

Figura 3.1: Árvore de contexto de um processo de renovação $\left(k_{0}=3\right)$

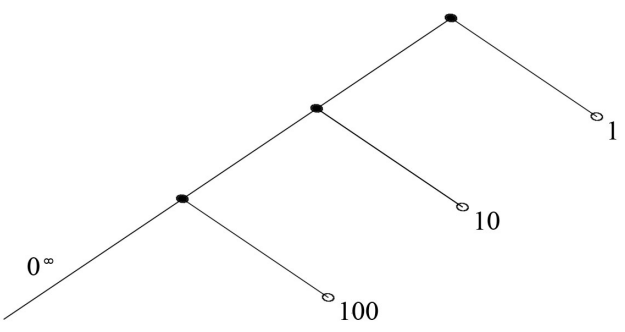

Figura 3.2: Árvore de contexto de um processo de renovação $\left(k_{0}=\infty\right)$

O objetivo deste capítulo é estudar as propriedades assintóticas dos estimadores de máxima verossimilhança $\hat{\tilde{\rho}}_{n}\left(x_{0}^{k-1}\right)$ e $\hat{\rho}_{n}\left(x_{0}^{k-1}\right)$, dados por

$$
\hat{\tilde{\rho}}_{n}\left(x_{0}^{k-1}\right)=\frac{N_{n}\left(x_{0}^{k-1} x_{0}^{k-1}\right)}{N_{n-k}\left(x_{0}^{k-1}\right)}
$$


e

$$
\hat{\rho}_{n}\left(x_{0}^{k-1}\right)=\frac{N_{n}\left(x_{0}^{k-1} x_{k-\tau\left(x_{0}^{k-1}\right)}^{k-1}\right)}{N_{n-\tau\left(x_{0}^{k-1}\right)}\left(x_{0}^{k-1}\right)} .
$$

Considere a função $N_{n}(y): \mathcal{A}^{\mathbb{N}} \rightarrow \mathbb{R}$, onde $y$ é uma sequência finita, definida da seguinte forma:

$$
N_{n}(y)=\sum_{j=0}^{n-l(y)} Y_{j}(y)
$$

onde

$$
Y_{j}(y)=\left\{\begin{array}{l}
1, \quad \text { se } \quad X_{j}^{j+l(y)-1}=y \\
0, \quad \text { caso contrário }
\end{array}\right.
$$

ou seja, $N_{n}(y)$ é a quantidade de vezes que a sequência $y$ aparece na amostra de tamanho $n$.

\subsection{Resultados obtidos para $\hat{\tilde{\rho}}_{n}\left(x_{0}^{k-1}\right)$}

Nesta seção mostraremos que a ocorrência dos eventos: uma amostra de tamanho $k-1$ aparecer um número mínimo de vezes e a distância entre $\tilde{\rho}_{n}\left(x_{0}^{k-1}\right)$ e o estimador $\hat{\tilde{\rho}}_{n}\left(x_{0}^{k-1}\right)$ ser superior a um erro tem probabilidade nula de ocorrer infinitas vezes.

Sejam $\xi$ e $\eta$ números reais positivos. Considere

$$
\epsilon\left(\xi, \eta, x_{0}^{k-1}\right)=\sqrt{\frac{\max \left\{2 \xi k, \eta \log \log N_{n-k}\left(x_{0}^{k-1}\right)\right\}}{N_{n-k}\left(x_{0}^{k-1}\right)}}
$$

e

$$
G_{n}\left(\xi, \eta, x_{0}^{k-1}\right)=\left\{\left|\hat{\tilde{\rho}}_{n}\left(x_{0}^{k-1}\right)-\mathbb{P}\left(X_{k}^{2 k-1}=x_{0}^{k-1} \mid X_{k-s}^{k-1}=x_{k-s}^{k-1}\right)\right|>\epsilon\left(\xi, \eta, x_{0}^{k-1}\right)\right\} .
$$

Seja $c$ uma constante positiva e

$$
H_{n}\left(c, x_{0}^{k-1}\right)=\left\{N_{n-k}\left(x_{0}^{k-1}\right)>2 c k\right\} .
$$

Além disso, considere $B_{n}\left(\xi, \eta, c, x_{0}^{k-1}\right)$ o evento $\left\{G_{n}\left(\xi, \eta, x_{0}^{k-1}\right) \cap H_{n}\left(c, x_{0}^{k-1}\right)\right\}$ e

$$
B_{n}=\bigcup_{k \geq s} \bigcup_{x_{0}^{k-1}} B_{n}\left(\xi, \eta, c, x_{0}^{k-1}\right)
$$

Fixando $\theta>1$, seja

$$
C_{m}\left(\xi, \eta, c, \theta, x_{0}^{k-1}\right)=\bigcup_{l=2 k}^{\infty}\left(B_{l}\left(\xi, \eta, c, x_{0}^{k-1}\right) \cap\left\{\theta^{m}<N_{l-k}\left(x_{0}^{k-1}\right) \leq \theta^{m+1}\right\}\right)
$$

$\mathrm{e}$

$$
C_{m}=\bigcup_{k \geq s} \bigcup_{x_{0}^{k-1}} C_{m}\left(\xi, \eta, c, \theta, x_{0}^{k-1}\right) .
$$

A seguir, apresentaremos um lema que será utilizado como ferramenta auxiliar na prova do teorema, que tem como objetivo mostrar que, sob determinadas condições,

$$
\mathbb{P}\left(\limsup B_{n} \backslash \lim \sup C_{m}\right)=0 .
$$


Observe que se, além desta condição,

$$
\mathbb{P}\left(\lim \sup C_{m}\right)=0
$$

também é válida, então podemos concluir que

$$
\mathbb{P}\left(\lim \sup B_{n}\right)=0 .
$$

Lema 3.7. Para $\xi, \eta$, c e $\theta$ fixos, tal que $\theta>1$, seja $B_{n}$ e $C_{m}$ como definidos em (3.4) e (3.5). Então temos que

$$
\mathbb{P}\left(\limsup B_{n} \backslash \lim \sup C_{m}\right)=0 .
$$

Prova. Seja $m_{k}=\left\lfloor\frac{\log (2 c k)}{\log \theta}\right\rfloor$ e considere que $\omega \in \lim \sup B_{n}$. Temos que

$$
\begin{aligned}
& \bigcup_{m=m_{k}}^{\infty} C_{m}\left(\xi, \eta, c, \theta, x_{0}^{k-1}\right)=\bigcup_{m=m_{k}}^{\infty} \bigcup_{j=2 k}^{\infty}\left(B_{j}\left(\xi, \eta, c, x_{0}^{k-1}\right) \cap\left\{\theta^{m}<N_{j-k}\left(x_{0}^{k-1}\right) \leq \theta^{m+1}\right\}\right) \\
& =\bigcup_{j=2 k}^{\infty}\left(B_{j}\left(\xi, \eta, c, x_{0}^{k-1}\right) \cap\left\{N_{j-k}\left(x_{0}^{k-1}\right)>\theta^{m_{k}}\right\}\right) . \\
& \text { Como } \frac{\log (2 c k)}{\log \theta}-1<m_{k}<\frac{\log (2 c k)}{\log \theta} \text {, temos que } \theta^{m_{k}}<2 c k<\theta^{m_{k}+1} \text {. Desta forma, } \\
& \bigcup_{j=2 k}^{\infty}\left(B_{j}\left(\xi, \eta, c, x_{0}^{k-1}\right) \cap\left\{N_{j-k}\left(x_{0}^{k-1}\right)>\theta^{m_{k}}\right\}\right) \supset \bigcup_{j=2 k}^{\infty}\left(B_{j}\left(\xi, \eta, c, x_{0}^{k-1}\right) \cap\left\{N_{j-k}\left(x_{0}^{k-1}\right)>2 c k\right\}\right) \\
& =\bigcup_{j=2 k}^{\infty} B_{j}\left(\xi, \eta, c, x_{0}^{k-1}\right) \supset B_{n}\left(\xi, \eta, c, x_{0}^{k-1}\right), \forall n \geq 2 k .
\end{aligned}
$$

Do enunciado, temos que $\omega \in \lim \sup B_{n}$, ou seja, existem infinitos $i$ tal que $\omega \in B_{n_{i}}\left(\xi, \eta, c, x(i)_{0}^{k_{n_{i}}-1}\right)$.

Como

$$
B_{n_{i}}\left(\xi, \eta, c, x(i)_{0}^{k_{n_{i}}-1}\right) \subset \bigcup_{m=m_{k_{n_{i}}}} C_{m}\left(\xi, \eta, c, \theta, x(i)_{0}^{k_{n_{i}}-1}\right),
$$

então

$$
B_{n_{i}}\left(\xi, \eta, c, x(i)_{0}^{k_{n_{i}}-1}\right) \subset C_{l_{n_{n_{i}}}}\left(\xi, \eta, c, \theta, x(i)_{0}^{k_{n_{i}}-1}\right)
$$

para algum $l_{k_{n_{i}}} \geq m_{k_{n_{i}}}$.

Portanto, existem infinitos $i$ tal que $\omega \in C_{l_{k_{n_{i}}}}\left(\xi, \eta, c, \theta, x(i)_{0}^{k_{n_{i}}-1}\right)$, para algum $l_{k_{n_{i}}} \geq m_{k_{n_{i}}}$. Desta forma, concluímos que $\omega \in \lim \sup C_{m}$.

Teorema 3.8. Considere $\left(X_{m}\right)_{m \in \mathbb{N}}$ um processo estocástico, ergódico, estacionário e compatível com a árvore de contexto probabilística $(\mathcal{T}, \mathbb{P})$. Para $s<k$, considere a sequência $x_{0}^{k-1}$ tal que $x_{k-s}^{k-1} \in \mathcal{T}$.Então, para todo $\xi>\frac{\log |A|}{2}$, existem $\eta>0$ e c $>0$ tal que

$$
\mathbb{P}\left(\left\{G_{n}\left(\xi, \eta, x_{0}^{k-1}\right) \cap H_{n}\left(c, x_{0}^{k-1}\right)\right\} i . v .\right)=0 .
$$

Prova. Seja $B_{n}\left(\xi, \eta, c, x_{0}^{k-1}\right)$ o evento $\left\{G_{n}\left(\xi, \eta, x_{0}^{k-1}\right) \cap H_{n}\left(c, x_{0}^{k-1}\right)\right\}$ e

$$
B_{n}=\bigcup_{k \geq s} \bigcup_{x_{0}^{k-1}} B_{n}\left(\xi, \eta, c, x_{0}^{k-1}\right)
$$


Iremos mostrar que para todo $\xi>\frac{\log |A|}{2}$ e $\eta$ e $c$ suficientemente grandes,

$$
\mathbb{P}\left(\lim \sup B_{n}\right)=0 \text {. }
$$

Fixando $\theta>1$, considere

$$
C_{m}\left(\xi, \eta, c, \theta, x_{0}^{k-1}\right)=\bigcup_{l=2 k}^{\infty}\left(B_{l}\left(\xi, \eta, c, x_{0}^{k-1}\right) \cap\left\{\theta^{m}<N_{l-k}\left(x_{0}^{k-1}\right) \leq \theta^{m+1}\right\}\right),
$$

e

$$
C_{m}=\bigcup_{k \geq s} \bigcup_{x_{0}^{k-1}} C_{m}\left(\xi, \eta, c, \theta, x_{0}^{k-1}\right) .
$$

Para mostrarmos que $\mathbb{P}\left(\lim \sup B_{n}\right)=0$, iremos utilizar o Lema 3.7. Conforme já mencionado, é suficiente mostrar que $\mathbb{P}\left(\lim \sup C_{m}\right)=0$, ou, por Borel-Cantelli, que

$$
\sum_{m=1}^{\infty} \mathbb{P}\left(C_{m}\right)<\infty
$$

para uma escolha adequada de $\theta>1$ na definição de $C_{m}$.

Considere

$$
Z_{n}= \begin{cases}N_{n}\left(x_{0}^{k-1} x_{0}^{k-1}\right)-\mathbb{P}\left(X_{k}^{2 k-1}=x_{0}^{k-1} \mid X_{k-s}^{k-1}=x_{k-s}^{k-1}\right) N_{n-k}\left(x_{0}^{k-1}\right), & \text { para } n \geq 2 k \\ 0, & \text { para } 0 \leq n<2 k\end{cases}
$$

Pelo Lema A.1, para todo $n \geq 2 k$, temos que $Z_{n}$ é um martingal e que $\left|Z_{n+1}-Z_{n}\right| \leq 1$.

Para todo $n \geq 2 k$, seja

$$
\begin{aligned}
u(n) & =\mathbb{1}\left\{X_{n-2 k}^{n-1}=x_{0}^{k-1} x_{0}^{k-1}\right\}-E\left[\mathbb{1}\left\{X_{n-2 k}^{n-1}=x_{0}^{k-1} x_{0}^{k-1}\right\} \mid \mathcal{F}_{n-k-1}\right] \\
& =\mathbb{1}\left\{X_{n-2 k}^{n-1}=x_{0}^{k-1} x_{0}^{k-1}\right\}-\mathbb{1}\left\{X_{n-2 k}^{n-k-1}=x_{0}^{k-1}\right\} \mathbb{P}\left(X_{k}^{2 k-1}=x_{0}^{k-1} \mid X_{k-s}^{k-1}=x_{k-s}^{k-1}\right) .
\end{aligned}
$$

Observe que $Z_{n}-Z_{n-1}=u(n)$. Assim, a sequência de variáveis aleatórias $A_{n}$, onde $\left(A_{m}\right)_{m \in \mathbb{N}}$ denota o processo crescente associado com o submartingal $\left(Z_{m}^{2}\right)_{m \in \mathbb{N}}$ pela Decomposição de Doob, é dado por

$$
A_{n}=\sum_{t=2 k}^{n} E\left(\left(Z_{t}-Z_{t-1}\right)^{2} \mid \mathcal{F}_{t-k-1}\right)=\sum_{t=2 k}^{n} E\left((u(t))^{2} \mid \mathcal{F}_{t-k-1}\right)
$$

Como,

$$
\begin{aligned}
u(n)^{2} & =\mathbb{1}\left\{X_{n-2 k}^{n-1}=x_{0}^{k-1} x_{0}^{k-1}\right\} \\
& -2 \mathbb{1}\left\{X_{n-2 k}^{n-1}=x_{0}^{k-1} x_{0}^{k-1}\right\} \mathbb{1}\left\{X_{n-2 k}^{n-k-1}=x_{0}^{k-1}\right\}\left[\mathbb{P}\left(X_{k}^{2 k-1}=x_{0}^{k-1} \mid X_{k-s}^{k-1}=x_{k-s}^{k-1}\right)\right] \\
& +\mathbb{1}\left\{X_{n-2 k}^{n-k-1}=x_{0}^{k-1}\right\}\left[\mathbb{P}\left(X_{k}^{2 k-1}=x_{0}^{k-1} \mid X_{k-s}^{k-1}=x_{k-s}^{k-1}\right)\right]^{2}
\end{aligned}
$$

temos que

$$
\begin{aligned}
E\left(u(n)^{2} \mid \mathcal{F}_{n-k-1}\right) & =\mathbb{1}\left\{X_{n-2 k}^{n-k-1}=x_{0}^{k-1}\right\}\left[\mathbb{P}\left(X_{k}^{2 k-1}=x_{0}^{k-1} \mid X_{k-s}^{k-1}=x_{k-s}^{k-1}\right)\right] \\
& -2 \mathbb{1}\left\{X_{n-2 k}^{n-k-1}=x_{0}^{k-1}\right\}\left[\mathbb{P}\left(X_{k}^{2 k-1}=x_{0}^{k-1} \mid X_{k-s}^{k-1}=x_{k-s}^{k-1}\right)\right]^{2} \\
& +\mathbb{1}\left\{X_{n-2 k}^{n-k-1}=x_{0}^{k-1}\right\}\left[\mathbb{P}\left(X_{k}^{2 k-1}=x_{0}^{k-1} \mid X_{k-s}^{k-1}=x_{k-s}^{k-1}\right)\right]^{2}
\end{aligned}
$$


Portanto,

$$
\begin{aligned}
E\left(u(n)^{2} \mid \mathcal{F}_{n-k-1}\right) & = \\
& =\mathbb{1}\left\{X_{n-2 k}^{n-k-1}=x_{0}^{k-1}\right\}\left[\mathbb{P}\left(X_{k}^{2 k-1}=x_{0}^{k-1} \mid X_{k-s}^{k-1}=x_{k-s}^{k-1}\right)\right]\left[1-\mathbb{P}\left(X_{k}^{2 k-1}=x_{0}^{k-1} \mid X_{k-s}^{k-1}=x_{k-s}^{k-1}\right)\right] .
\end{aligned}
$$

Assim,

$$
\begin{aligned}
A_{n} & =\sum_{t=2 k}^{n} E\left(u(t)^{2} \mid \mathcal{F}_{t-k-1}\right) \\
& =\sum_{t=2 k}^{n} \mathbb{1}\left\{X_{t-2 k}^{t-k-1}=x_{0}^{k-1}\right\} \mathbb{P}\left(X_{k}^{2 k-1}=x_{0}^{k-1} \mid X_{k-s}^{k-1}=x_{k-s}^{k-1}\right)\left[1-\mathbb{P}\left(X_{k}^{2 k-1}=x_{0}^{k-1} \mid X_{k-s}^{k-1}=x_{k-s}^{k-1}\right)\right] \\
& =N_{n-k}\left(x_{0}^{k-1}\right) \mathbb{P}\left(X_{k}^{2 k-1}=x_{0}^{k-1} \mid X_{k-s}^{k-1}=x_{k-s}^{k-1}\right)\left[1-\mathbb{P}\left(X_{k}^{2 k-1}=x_{0}^{k-1} \mid X_{k-s}^{k-1}=x_{k-s}^{k-1}\right)\right] \leq \frac{1}{4} N_{n-k}\left(x_{0}^{k-1}\right) .
\end{aligned}
$$

Agora, fixe $\epsilon>0$. Considere

$$
f(\lambda)=\frac{\exp (\lambda)-1-\lambda}{\lambda}
$$

$\mathrm{e}$

$$
g(\lambda)=\left(\frac{1}{2}+\epsilon\right) \lambda
$$

Cálculos diretos mostram que $f(\lambda)=g(\lambda)$ tem uma única solução, que chamamos $\lambda_{0}(\epsilon)$.

Para $0<\lambda<\lambda_{0}(\epsilon)$ temos $f(\lambda)<g(\lambda)$ e para $\lambda>\lambda_{0}(\epsilon)$ temos $f(\lambda)>g(\lambda)$. Desta forma, para $0<\lambda<\lambda_{0}(\epsilon)$

$$
\left\{X>\left(\frac{1}{2}+\epsilon\right) \lambda\right\} \subset\left\{X>\frac{\exp (\lambda)-1-\lambda}{\lambda}\right\}
$$

Portanto, para todo $m \geq 0$ e $0<\lambda<\lambda_{0}(\epsilon)$, por (3.9) e (3.10) temos que

$$
\left\{\theta^{m}<N_{n-k}\left(x_{0}^{k-1}\right) \leq \theta^{m+1}\right\} \cap\left\{\left|Z_{n}\right|>\alpha+\left(\frac{1}{2}+\epsilon\right) \lambda \frac{\theta^{m+1}}{4}\right\} \subset\left\{\left|Z_{n}\right|>\alpha+\frac{\exp (\lambda)-1-\lambda}{\lambda} A_{n}\right\} .
$$

Iremos considerar $\alpha$ e $\lambda$ como

$$
\alpha(m)=\frac{\theta^{m / 2} \sqrt{\max [2 \xi k, \eta \log (m \log \theta)]}}{2(1+\epsilon)}
$$

e

$$
\lambda(m)=\frac{4 \sqrt{\max [2 \xi k, \eta \log (m \log \theta)]}}{(1+\epsilon) \theta^{1+m / 2}} .
$$

Para garantir que $\lambda<\lambda_{0}(\epsilon)$, necessário em (3.11), assumimos que $m$ satisfaz

$$
\theta^{m+1}>\tau(\epsilon) \max [2 \xi k, \eta \log (m \log \theta)] \quad \text { onde } \quad \tau(\epsilon)=\left(\frac{4}{\lambda_{0}(\epsilon)}\right)^{2}
$$

e também excede um patamar $m_{0}(\epsilon, \theta, \eta)$.

Considerando $\theta^{m}<N_{n-k}\left(x_{0}^{k-1}\right)$, temos que

$$
\begin{aligned}
\alpha+\left(\frac{1}{2}+\epsilon\right) \lambda \frac{\theta^{m+1}}{4} & =\theta^{m / 2} \sqrt{\max [2 \xi k, \eta \log (m \log \theta)]} \\
& <\sqrt{N_{n-k}\left(x_{0}^{k-1}\right) \max \left[2 \xi k, \eta \log \log \left(N_{n-k}\left(x_{0}^{k-1}\right)\right)\right]} .
\end{aligned}
$$


Além disso,

$$
\alpha \lambda=\mu \max [2 \xi k, \eta \log (m \log \theta)]
$$

onde

$$
\mu=\frac{2}{(1+\epsilon)^{2} \theta}
$$

Assim, por (3.11), (3.12) e pelo Corolário A.10,

$$
\begin{aligned}
& \mathbb{P}\left(\bigcup_{n=2 k}^{\infty}\left(\theta^{m}<N_{n-k}\left(x_{0}^{k-1}\right) \leq \theta^{m+1}\right) \cap\left(\left|Z_{n}\right|>\sqrt{N_{n-k}\left(x_{0}^{k-1}\right) \max \left[2 \xi k, \eta \log \log N_{n-k}\left(x_{0}^{k-1}\right)\right]}\right)\right) \\
& <\mathbb{P}\left(\bigcup_{n=2 k}^{\infty}\left(\theta^{m}<N_{n-k}\left(x_{0}^{k-1}\right) \leq \theta^{m+1}\right) \cap\left(\left|Z_{n}\right|>\alpha+\left(\frac{1}{2}+\epsilon\right) \lambda \frac{\theta^{m+1}}{4}\right)\right) \\
& <\mathbb{P}\left(\bigcup_{n=2 k}^{\infty}\left(\left|Z_{n}\right|>\alpha+\frac{\exp (\lambda)-1-\lambda}{\lambda} A_{n}\right)\right) \leq 2 \exp (-\lambda \alpha),
\end{aligned}
$$

onde $2 \exp (-\lambda \alpha)=2 \exp (-\mu \max [2 \xi k, \eta \log (m \log \theta)])$.

Note que a desigualdade (3.13) é válida quando $0<\lambda<\lambda_{0}(\epsilon)$, isto é, quando $m$ satisfaz $\theta^{m+1}>\tau(\epsilon) \max [2 \xi k, \eta \log (m \log \theta)], \operatorname{com} \tau(\epsilon)=\left(\frac{4}{\lambda_{0}(\epsilon)}\right)^{2}$ e quando $m>m_{0}(\epsilon, \theta, \eta)$.

Da equação (3.8), para $n \geq 2 k$, observamos que

$$
\begin{aligned}
\frac{Z_{n}}{N_{n-k}\left(x_{0}^{k-1}\right)} & =\frac{N_{n}\left(x_{0}^{k-1} x_{0}^{k-1}\right)}{N_{n-k}\left(x_{0}^{k-1}\right)}-\frac{\mathbb{P}\left(X_{k}^{2 k-1}=x_{0}^{k-1} \mid X_{k-s}^{k-1}=x_{k-s}^{k-1}\right) N_{n-k}\left(x_{0}^{k-1}\right)}{N_{n-k}\left(x_{0}^{k-1}\right)} \\
& =\hat{\tilde{\rho}}_{n}\left(x_{0}^{k-1}\right)-\mathbb{P}\left(X_{k}^{2 k-1}=x_{0}^{k-1} \mid X_{k-s}^{k-1}=x_{k-s}^{k-1}\right)
\end{aligned}
$$

e temos que $C_{m}\left(\xi, \eta, c, \theta, x_{0}^{k-1}\right)$ é igual a intersecção do evento

$$
\left\{\bigcup_{n=2 k}^{\infty}\left(\theta^{m}<N_{n-k}\left(x_{0}^{k-1}\right) \leq \theta^{m+1}\right) \cap\left(\left|Z_{n}\right|>\sqrt{N_{n-k}\left(x_{0}^{k-1}\right) \max \left[2 \xi k, \eta \log \log N_{n-k}\left(x_{0}^{k-1}\right)\right]}\right)\right\}
$$

com o evento

$$
\left\{N_{n-k}\left(x_{0}^{k-1}\right)>2 c k\right\} .
$$

Quando a intersecção é não vazia, temos que $\theta^{m+1}>\tau(\epsilon) \max [2 \xi k, \eta \log (m \log \theta)]$ e como $\theta^{m+1} \geq$ $N_{n-k}\left(x_{0}^{k-1}\right)>2 c k$, temos que $2 c k \geq \tau(\epsilon) \max [2 \xi k, \eta \log (m \log \theta)]$.

Assim, se $c$ em (3.3) é suficientemente grande, então para todo $m>m_{0}(\epsilon, \theta, \eta)$ e para todo $x_{0}^{k-1}$ com $k>s$ :

$$
\begin{aligned}
& \mathbb{P}\left(C_{m}\left(\xi, \eta, c, \theta, x_{0}^{k-1}\right)\right) \leq 2 \exp (-\mu \max [2 \xi k, \eta \log (m \log \theta)]) \\
& = \begin{cases}2 \exp (-2 \mu \xi k), & \text { se } k \geq \frac{\eta}{2 \xi} \log (m \log \theta) \\
2(m \log \theta)^{-\mu \eta}, & \text { se } k<\frac{\eta}{2 \xi} \log (m \log \theta) .\end{cases}
\end{aligned}
$$

Assuma $\theta$ e $\epsilon$ até agora arbitrários são selecionados tais que $\mu \xi=\frac{2 \xi}{(1+\epsilon)^{2} \theta}>\log |A|$, que é 
possível pela suposição $\xi>\frac{\log |A|}{2}$. De (3.7), temos que

$$
\mathbb{P}\left(C_{m}\right)=\mathbb{P}\left(\bigcup_{k \geq s} \bigcup_{x_{0}^{k-1}} C_{m}\left(\xi, \eta, c, \theta, x_{0}^{k-1}\right)\right) \leq \sum_{k \geq s} \sum_{x_{0}^{k-1}} \mathbb{P}\left(C_{m}\left(x_{0}^{k-1}\right)\right)
$$

Utilizando a desigualdade (3.14),

$$
\begin{aligned}
\sum_{k \geq s} \sum_{x_{0}^{k-1}} \mathbb{P}\left(C_{m}\left(\xi, \eta, c, \theta, x_{0}^{k-1}\right)\right) & \leq 2\left[\sum_{k \geq \frac{\eta}{2 \xi} \log (m \log \theta)} \exp (-2 \mu \xi k)\left\{\# x_{0}^{k-1}: k \geq \frac{\eta}{2 \xi} \log (m \log \theta)\right\}\right] \\
& +2\left[\sum_{k<\frac{\eta}{2 \xi} \log (m \log \theta)}(m \log \theta)^{-\mu \eta}\left\{\# x_{0}^{k-1}: k<\frac{\eta}{2 \xi} \log (m \log \theta)\right\}\right] \\
& =2\left[\sum_{k \geq \frac{\eta}{2 \xi} \log (m \log \theta)}|A|^{k} \exp (-2 \mu \xi k)+\left(\sum_{k<\frac{\eta}{2 \xi} \log (m \log \theta)}|A|^{k}\right)(m \log \theta)^{-\mu \eta}\right] .
\end{aligned}
$$

Para o cálculo do primeiro termo do lado direito de (3.15), observe que

$$
\begin{aligned}
\sum_{k \geq \frac{\eta}{2 \xi} \log (m \log \theta)}^{\infty}|A|^{k} \exp (-2 \mu \xi k) & =\sum_{k \geq \frac{\eta}{2 \xi} \log (m \log \theta)}^{\infty} \exp (-k(2 \mu \xi-\log |A|)) \\
& =\frac{\exp \left(-\frac{\eta}{2 \xi} \log (m \log \theta)(2 \mu \xi-\log |A|)\right)}{1-\exp (-(2 \mu \xi-\log |A|))}
\end{aligned}
$$

Para o segundo termo do lado direito da equação (3.15), temos que

$$
\left(\sum_{k<\frac{\eta}{2 \xi} \log (m \log \theta)}|A|^{k}\right)(m \log \theta)^{-\mu \eta} \leq(m \log \theta)^{-\mu \eta}|A|^{\frac{\eta}{2 \xi} \log (m \log \theta)+1}
$$

Como

$$
|A|^{\frac{\eta}{2 \xi} \log (m \log \theta)}=(m \log \theta)^{\frac{\eta}{2 \xi} \log |A|},
$$

então temos por (3.16), (3.17) e (3.18),

$$
\begin{aligned}
\mathbb{P}\left(C_{m}\right) & \leq 2\left(\frac{\exp \left(-\frac{\eta}{2 \xi} \log (m \log \theta)(2 \mu \xi-\log |A|)\right)}{1-\exp (-(2 \mu \xi-\log |A|))}+(m \log \theta)^{-\mu \eta}|A|(m \log \theta)^{\frac{\eta}{2 \xi} \log |A|}\right) \\
& =2\left(\frac{(m \log \theta)^{-\eta \mu+\frac{\eta \log |A|}{2 \xi}}}{1-\exp (-(2 \mu \xi-\log |A|))}+|A|(m \log \theta)^{-\eta \mu+\frac{\eta \log |A|}{2 \xi}}\right) \\
& =\pi \times(m \log \theta)^{-\left(\mu-\frac{\log |A|}{2 \xi}\right) \eta},
\end{aligned}
$$

onde $\pi$ é constante.

Portanto, se $\eta>\frac{2 \xi}{2 \mu \xi-\log |A|}$, temos que $\sum_{m=1}^{\infty} \mathbb{P}\left(C_{m}\right)<\infty$, ou seja, $\mathbb{P}\left(\lim \sup C_{m}\right)=0$. 


\subsubsection{Convergência do estimador $\hat{\tilde{\rho}}_{n}\left(x_{0}^{k-1}\right)$}

A seguir verificaremos que, para uma sequência fixa $x_{0}^{k-1}$, pelo Lema A.11 e como consequência do Teorema 3.8, a distância entre a probabilidade condicional $\mathbb{P}\left(X_{k}^{2 k-1}=x_{0}^{k-1} \mid X_{k-s}^{k-1}=x_{k-s}^{k-1}\right)$ e seu estimador $\hat{\tilde{\rho}}_{n}\left(x_{0}^{k-1}\right)$ converge para zero.

Corolário 3.9. Considere os conjuntos $G_{n}\left(\xi, \eta, x_{0}^{k-1}\right)$ e $H_{n}\left(c, x_{0}^{k-1}\right)$ definidos em (3.2) e (3.3). Sob as hipóteses do Teorema 3.8, temos que

$$
\mathbb{P}\left(\left\{G_{n}\left(\xi, \eta, x_{0}^{k-1}\right)\right\} i . v .\right)=0 .
$$

Prova. Considere $G_{n}\left(\xi, \eta, x_{0}^{k-1}\right)$ e $H_{n}\left(c, x_{0}^{k-1}\right)$ definidos em (3.2) e (3.3), respectivamente. Pelo Teorema 3.8 , obtemos o seguinte resultado

$$
\mathbb{P}\left(\left\{G_{n}\left(\xi, \eta, x_{0}^{k-1}\right) \cap H_{n}\left(c, x_{0}^{k-1}\right)\right\} i . v .\right)=0 .
$$

Observe também que $H_{n}\left(c, x_{0}^{k-1}\right)$ é um conjunto crescente e existe um número inteiro positivo $n_{0}$ tal que

$$
N_{n-k}\left(x_{0}^{k-1}\right)>2 c k,
$$

para todo $n>n_{0}$.Logo,

$$
\mathbb{P}\left(\left\{H_{n}\left(c, x_{0}^{k-1}\right)\right\} i . v .\right)=1
$$

Portanto, pelo Lema A.11 podemos concluir, sob as condições do Teorema 3.8, que

$$
\mathbb{P}\left(\left\{G_{n}\left(\xi, \eta, x_{0}^{k-1}\right)\right\} i . v .\right)=0 .
$$

Logo,

$$
\mathbb{P}\left(\left\{\left|\hat{\tilde{\rho}}_{n}\left(x_{0}^{k-1}\right)-\mathbb{P}\left(X_{k}^{2 k-1}=x_{0}^{k-1} \mid X_{k-s}^{k-1}=x_{k-s}^{k-1}\right)\right|>\epsilon\left(\xi, \eta, x_{0}^{k-1}\right)\right\} i . v .\right)=0,
$$

e,

$$
\hat{\tilde{\rho}}_{n}\left(x_{0}^{k-1}\right) \underset{n \rightarrow \infty}{\stackrel{\text { q.c. }}{\longrightarrow}} \mathbb{P}\left(X_{k}^{2 k-1}=x_{0}^{k-1} \mid X_{k-s}^{k-1}=x_{k-s}^{k-1}\right) .
$$

Na próxima seção será apresentado o teorema e o corolário quando consideramos $\rho_{n}\left(x_{0}^{k-1}\right)$ e o respectivo estimador $\hat{\rho}_{n}\left(x_{0}^{k-1}\right)$.

\subsection{Resultados obtidos para $\hat{\rho}_{n}\left(x_{0}^{k-1}\right)$}

Nesta seção mostraremos que a ocorrência dos eventos: uma amostra de tamanho $k-1$ aparecer um número mínimo de vezes e a distância entre o estimador $\rho_{n}\left(x_{0}^{k-1}\right)$ e o valor estimado $\hat{\rho}_{n}\left(x_{0}^{k-1}\right)$ ser superior a um erro tem probabilidade nula de ocorrer infinitas vezes.

Sejam $\xi$ e $\eta$ números reais positivos. Considere

$$
\epsilon\left(\xi, \eta, x_{0}^{k-1}\right)=\sqrt{\frac{\max \left\{2 \xi k, \eta \log \log N_{n-\tau\left(x_{0}^{k-1}\right)}\left(x_{0}^{k-1}\right)\right\}}{N_{n-\tau\left(x_{0}^{k-1}\right)}\left(x_{0}^{k-1}\right)}} .
$$

e

$$
G_{n}\left(\xi, \eta, x_{0}^{k-1}\right)=\left\{\left|\hat{\rho}_{n}\left(x_{0}^{k-1}\right)-\mathbb{P}\left(X_{k}^{k+\tau\left(x_{0}^{k-1}\right)-1}=x_{k-\tau\left(x_{0}^{k-1}\right)}^{k-1} \mid X_{k-s}^{k-1}=x_{k-s}^{k-1}\right)\right|>\epsilon\left(\xi, \eta, x_{0}^{k-1}\right)\right\} .
$$


Seja $c$ uma constante positiva e

$$
H_{n}\left(c, x_{0}^{k-1}\right)=\left\{N_{n-\tau\left(x_{0}^{k-1}\right)}\left(x_{0}^{k-1}\right)>2 c k\right\} .
$$

Além disso, considere $B_{n}\left(\xi, \eta, c, x_{0}^{k-1}\right)$ o evento $\left\{G_{n}\left(\xi, \eta, x_{0}^{k-1}\right) \cap H_{n}\left(c, x_{0}^{k-1}\right)\right\}$ e

$$
B_{n}=\bigcup_{k \geq s} \bigcup_{x_{0}^{k-1}} B_{n}\left(\xi, \eta, c, x_{0}^{k-1}\right)
$$

Fixando $\theta>1$, considere

$$
C_{m}\left(\xi, \eta, c, \theta, x_{0}^{k-1}\right)=\bigcup_{l=k+\tau\left(x_{0}^{k-1}\right)}^{\infty}\left(B_{l}\left(\xi, \eta, c, x_{0}^{k-1}\right) \cap\left\{\theta^{m}<N_{l-\tau\left(x_{0}^{k-1}\right)}\left(x_{0}^{k-1}\right) \leq \theta^{m+1}\right\}\right),
$$

e

$$
C_{m}=\bigcup_{k \geq s} \bigcup_{x_{0}^{k-1}} C_{m}\left(\xi, \eta, c, \theta, x_{0}^{k-1}\right)
$$

A seguir, apresentaremos um lema que será utilizado como ferramenta auxiliar na prova do teorema que, sob determinadas condições,

$$
\mathbb{P}\left(\lim \sup B_{n} \backslash \lim \sup C_{m}\right)=0 .
$$

Observe que se, além desta condição,

$$
\mathbb{P}\left(\lim \sup C_{m}\right)=0
$$

também é válida, então podemos concluir que

$$
\mathbb{P}\left(\lim \sup B_{n}\right)=0 .
$$

Lema 3.10. Para $\xi, \eta$, c e $\theta$ fixos, tal que $\theta>1$, seja $B_{n}$ e $C_{m}$ como definidos em (3.23) e (3.24). Então temos que

$$
\mathbb{P}\left(\lim \sup B_{n} \backslash \lim \sup C_{m}\right)=0 .
$$

Prova. Seja $m_{k}=\left\lfloor\frac{\log (2 c k)}{\log \theta}\right\rfloor$ e considere que $\omega \in \lim \sup B_{n}$. Temos que

$$
\begin{aligned}
\bigcup_{m=m_{k}}^{\infty} C_{m}\left(\xi, \eta, c, \theta, x_{0}^{k-1}\right) & =\bigcup_{m=m_{k}}^{\infty} \bigcup_{j=k+\tau\left(x_{0}^{k-1}\right)}^{\infty}\left(B_{j}\left(\xi, \eta, c, x_{0}^{k-1}\right) \cap\left\{\theta^{m}<N_{j-\tau\left(x_{0}^{k-1}\right)}\left(x_{0}^{k-1}\right) \leq \theta^{m+1}\right\}\right) \\
& =\bigcup_{j=k+\tau\left(x_{0}^{k-1}\right)}^{\infty}\left(B_{j}\left(\xi, \eta, c, x_{0}^{k-1}\right) \cap\left\{N_{j-\tau\left(x_{0}^{k-1}\right)}\left(x_{0}^{k-1}\right)>\theta^{m_{k}}\right\}\right) .
\end{aligned}
$$


Como $\frac{\log (2 c k)}{\log \theta}-1<m_{k}<\frac{\log (2 c k)}{\log \theta}$, temos que $\theta^{m_{k}}<2 c k<\theta^{m_{k}+1}$. Desta forma, $\bigcup_{j=k+\tau\left(x_{0}^{k-1}\right)}^{\infty}\left(B_{j}\left(\xi, \eta, c, x_{0}^{k-1}\right) \cap\left\{N_{j-\tau\left(x_{0}^{k-1}\right)}\left(x_{0}^{k-1}\right)>\theta^{m_{k}}\right\}\right)$

$$
\begin{aligned}
& \supset \bigcup_{j=k+\tau\left(x_{0}^{k-1}\right)}^{\infty}\left(B_{j}\left(\xi, \eta, c, x_{0}^{k-1}\right) \cap\left\{N_{j-\tau\left(x_{0}^{k-1}\right)}\left(x_{0}^{k-1}\right)>2 c k\right\}\right) \\
& =\bigcup_{j=k+\tau\left(x_{0}^{k-1}\right)}^{\infty} B_{j}\left(\xi, \eta, c, x_{0}^{k-1}\right) \supset B_{n}\left(\xi, \eta, c, x_{0}^{k-1}\right), \forall n \geq k+\tau\left(x_{0}^{k-1}\right) .
\end{aligned}
$$

Do enunciado, temos que $\omega \in \lim \sup B_{n}$, ou seja, existem infinitos $i$ tal que $\omega \in B_{n_{i}}\left(\xi, \eta, c, x(i)_{0}^{k_{n_{i}}-1}\right)$. Como

$$
B_{n_{i}}\left(\xi, \eta, c, x(i)_{0}^{k_{n_{i}}-1}\right) \subset \bigcup_{m=m_{k_{n_{i}}}} C_{m}\left(\xi, \eta, c, \theta, x(i)_{0}^{k_{n_{i}}-1}\right),
$$

então

$$
B_{n_{i}}\left(\xi, \eta, c, x(i)_{0}^{k_{n_{i}}-1}\right) \subset C_{l_{n_{n_{i}}}}\left(\xi, \eta, c, \theta, x(i)_{0}^{k_{n_{i}}-1}\right)
$$

para algum $l_{k_{n_{i}}} \geq m_{k_{n_{i}}}$.

Portanto, existem infinitos $i$ tal que $\omega \in C_{l_{k_{n_{i}}}}\left(\xi, \eta, c, \theta, x(i)_{0}^{k_{n_{i}}-1}\right)$, para algum $l_{k_{n_{i}}} \geq m_{k_{n_{i}}}$. Desta forma, concluímos que $\omega \in \lim \sup C_{m}$.

Teorema 3.11. Considere $\left(X_{m}\right)_{m \in \mathbb{N}}$ um processo estocástico, ergódico, estacionário e compativel com a árvore de contexto probabilística $(\mathcal{T}, \mathbb{P})$. Para $s<k$, considere a sequência $x_{0}^{k-1}$ tal que $x_{k-s}^{k-1} \in \mathcal{T}$.Então, para todo $\xi>\frac{\log |A|}{2}$, existem $\eta>0$ e c $>0$ tal que

$$
\mathbb{P}\left(\left\{G_{n}\left(\xi, \eta, x_{0}^{k-1}\right) \cap H_{n}\left(c, x_{0}^{k-1}\right)\right\} i . v .\right)=0 .
$$

Prova. Seja $B_{n}\left(\xi, \eta, c, x_{0}^{k-1}\right)$ o evento $\left\{G_{n}\left(\xi, \eta, x_{0}^{k-1}\right) \cap H_{n}\left(c, x_{0}^{k-1}\right)\right\}$ e

$$
B_{n}=\bigcup_{k \geq s} \bigcup_{x_{0}^{k-1}} B_{n}\left(\xi, \eta, c, x_{0}^{k-1}\right) .
$$

Iremos mostrar que para todo $\xi>\frac{\log |A|}{2}$ e $\eta$ e $c$ suficientemente grandes,

$$
\mathbb{P}\left(\lim \sup B_{n}\right)=0 \text {. }
$$

Fixando $\theta>1$, considere

$$
C_{m}\left(\xi, \eta, c, \theta, x_{0}^{k-1}\right)=\bigcup_{l=k+\tau\left(x_{0}^{k-1}\right)}^{\infty}\left(B_{l}\left(\xi, \eta, c, x_{0}^{k-1}\right) \cap\left\{\theta^{m}<N_{l-\tau\left(x_{0}^{k-1}\right)}\left(x_{0}^{k-1}\right) \leq \theta^{m+1}\right\}\right),
$$

e

$$
C_{m}=\bigcup_{k \geq s} \bigcup_{x_{0}^{k-1}} C_{m}\left(\xi, \eta, c, \theta, x_{0}^{k-1}\right) .
$$

Para mostrarmos que $\mathbb{P}\left(\lim \sup B_{n}\right)=0$, iremos utilizar o Lema 3.10. Conforme já mencionado, é suficiente mostrar que $\mathbb{P}\left(\lim \sup C_{m}\right)=0$, ou, por Borel-Cantelli, que 


$$
\sum_{m=1}^{\infty} \mathbb{P}\left(C_{m}\right)<\infty
$$

para uma escolha adequada de $\theta>1$ na definição de $C_{m}$.

Considere

$$
Z_{n}=\left\{\begin{array}{l}
N_{n}\left(x_{0}^{k-1} x_{k-\tau\left(x_{0}^{k-1}\right)}^{k-1}\right)-\mathbb{P}\left(X_{k}^{k+\tau\left(x_{0}^{k-1}\right)-1}=x_{k-\tau\left(x_{0}^{k-1}\right)}^{k-1} \mid X_{k-s}^{k-1}=x_{k-s}^{k-1}\right) N_{n-\tau\left(x_{0}^{k-1}\right)}\left(x_{0}^{k-1}\right), \text { para } n \geq k+\tau\left(x_{0}^{k-1}\right) \\
0, \text { para } 0 \leq n<k+\tau\left(x_{0}^{k-1}\right) .
\end{array}\right.
$$

Pelo Lema A.2, para todo $n \geq k+\tau\left(x_{0}^{k-1}\right)$, temos que $Z_{n}$ é um martingal e que $\left|Z_{n+1}-Z_{n}\right| \leq 1$.

Para todo $n \geq k+\tau\left(x_{0}^{k-1}\right)$, seja

$$
\begin{aligned}
u(n) & =\mathbb{1}\left\{X_{n-k-\tau\left(x_{0}^{k-1}\right)}^{n-1}=x_{0}^{k-1} x_{k-\tau\left(x_{0}^{k-1}\right)}^{k-1}\right\}-E\left[\mathbb{1}\left\{X_{n-k-\tau\left(x_{0}^{k-1}\right)}^{n-1}=x_{0}^{k-1} x_{k-\tau\left(x_{0}^{k-1}\right)}^{k-1}\right\} \mid \mathcal{F}_{n-\tau\left(x_{0}^{k-1}\right)-1}\right] \\
& =\mathbb{1}\left\{X_{n-k-\tau\left(x_{0}^{k-1}\right)}^{n-1}=x_{0}^{k-1} x_{k-\tau\left(x_{0}^{k-1}\right)}^{k-1}\right\}-\mathbb{1}\left\{X_{n-k-\tau\left(x_{0}^{k-1}\right)}^{n-\tau\left(x_{0}^{k-1}\right)-1}=x_{0}^{k-1}\right\} \mathbb{P}\left(X_{k}^{k+\tau\left(x_{0}^{k-1}\right)-1}=x_{k-\tau\left(x_{0}^{k-1}\right)}^{k-1} \mid X_{k-s}^{k-1}=x_{k-s}^{k-1}\right)
\end{aligned}
$$

Observe que $Z_{n}-Z_{n-1}=u(n)$. Assim, a sequência de variáveis aleatórias $A_{n}$, onde $\left(A_{m}\right)_{m \in \mathbb{N}}$ denota o processo crescente associado com o submartingal $\left(Z_{m}^{2}\right)_{m \in \mathbb{N}}$ pela Decomposição de Doob, é dado por

$$
A_{n}=\sum_{t=k+\tau\left(x_{0}^{k-1}\right)}^{n} E\left(\left(Z_{t}-Z_{t-1}\right)^{2} \mid \mathcal{F}_{t-\tau\left(x_{0}^{k-1}\right)-1}\right)=\sum_{t=k+\tau\left(x_{0}^{k-1}\right)}^{n} E\left((u(t))^{2} \mid \mathcal{F}_{t-\tau\left(x_{0}^{k-1}\right)-1}\right) .
$$

Como,

$$
\begin{aligned}
u(n)^{2} & =\mathbb{1}\left\{X_{n-k-\tau\left(x_{0}^{k-1}\right)}^{n-1}=x_{0}^{k-1} x_{k-\tau\left(x_{0}^{k-1}\right)}^{k-1}\right\} \\
& -2 \mathbb{1}\left\{X_{n-k-\tau\left(x_{0}^{k-1}\right)}^{n-1}=x_{0}^{k-1} x_{k-\tau\left(x_{0}^{k-1}\right)}^{k-1}\right\} \mathbb{1}\left\{X_{n-k-\tau\left(x_{0}^{k-1}\right)}^{n-\tau\left(x_{k}^{k-1}\right)-1}=x_{0}^{k-1}\right\}\left[\mathbb{P}\left(X_{k}^{k+\tau\left(x_{0}^{k-1}\right)-1}=x_{k-\tau\left(x_{0}^{k-1}\right)}^{k-1} \mid X_{k-s}^{k-1}=x_{k-s}^{k-1}\right)\right. \\
& +\mathbb{1}\left\{X_{n-k-\tau\left(x_{0}^{k-1}\right)}^{n-\tau\left(x_{k}^{k-1}\right)-1}=x_{0}^{k-1}\right\}\left[\mathbb{P}\left(X_{k}^{k+\tau\left(x_{0}^{k-1}\right)-1}=x_{k-\tau\left(x_{0}^{k-1}\right)}^{k-1} \mid X_{k-s}^{k-1}=x_{k-s}^{k-1}\right)\right]^{2}
\end{aligned}
$$

temos que

$$
\begin{aligned}
E\left(u(n)^{2} \mid \mathcal{F}_{n-\tau\left(x_{0}^{k-1}\right)-1}\right) & =\mathbb{1}\left\{X_{n-k-\tau\left(x_{0}^{k-1}\right)}^{n-\tau\left(x_{0}^{k-1}\right)-1}=x_{0}^{k-1}\right\}\left[\mathbb{P}\left(X_{k}^{k+\tau\left(x_{0}^{k-1}\right)-1}=x_{k-\tau\left(x_{0}^{k-1}\right)}^{k-1} \mid X_{k-s}^{k-1}=x_{k-s}^{k-1}\right)\right] \\
& -2 \mathbb{1}\left\{X_{n-k-\tau\left(x_{0}^{k-1}\right)}^{n-\tau\left(x_{0}^{k-1}\right)-1}=x_{0}^{k-1}\right\}\left[\mathbb{P}\left(X_{k}^{k+\tau\left(x_{0}^{k-1}\right)-1}=x_{k-\tau\left(x_{0}^{k-1}\right)}^{k-1} \mid X_{k-s}^{k-1}=x_{k-s}^{k-1}\right)\right]^{2} \\
& +\mathbb{1}\left\{X_{n-k-\tau\left(x_{0}^{k-1}\right)}^{n-\tau\left(x_{0}^{k-1}\right)-1}=x_{0}^{k-1}\right\}\left[\mathbb{P}\left(X_{k}^{k+\tau\left(x_{0}^{k-1}\right)-1}=x_{k-\tau\left(x_{0}^{k-1}\right)}^{k-1} \mid X_{k-s}^{k-1}=x_{k-s}^{k-1}\right)\right]^{2} .
\end{aligned}
$$

Portanto,

$$
\begin{aligned}
E\left(u(n)^{2} \mid \mathcal{F}_{n-\tau\left(x_{0}^{k-1}\right)-1}\right) & = \\
& =\mathbb{1}\left\{X_{n-k-\tau\left(x_{0}^{k-1}\right)}^{n-\tau\left(x_{k-1}^{k-1}\right)-1}=x_{0}^{k-1}\right\}\left[\mathbb{P}\left(X_{k}^{k+\tau\left(x_{0}^{k-1}\right)-1}=x_{k-\tau\left(x_{0}^{k-1}\right)}^{k-1} \mid X_{k-s}^{k-1}=x_{k-s}^{k-1}\right)\right] \\
& {\left[1-\mathbb{P}\left(X_{k}^{k+\tau\left(x_{0}^{k-1}\right)-1}=x_{k-\tau\left(x_{0}^{k-1}\right)}^{k-1} \mid X_{k-s}^{k-1}=x_{k-s}^{k-1}\right)\right] . }
\end{aligned}
$$


Assim,

$$
\begin{aligned}
A_{n} & =\sum_{t=k+\tau\left(x_{0}^{k-1}\right)}^{n} E\left(u(t)^{2} \mid \mathcal{F}_{t-\tau\left(x_{0}^{k-1}\right)-1}\right) \\
& =\sum_{t=k+\tau\left(x_{0}^{k-1}\right)}^{n}\left\{X_{t-k-\tau\left(x_{0}^{k-1}\right)}^{t-\tau\left(x_{k}^{k-1}\right)-1}=x_{0}^{k-1}\right\} \mathbb{P}\left(X_{k}^{k+\tau\left(x_{0}^{k-1}\right)-1}=x_{k-\tau\left(x_{0}^{k-1}\right)}^{k-1} \mid X_{k-s}^{k-1}=x_{k-s}^{k-1}\right) \\
& {\left[1-\mathbb{P}\left(X_{k}^{k+\tau\left(x_{0}^{k-1}\right)-1}=x_{k-\tau\left(x_{0}^{k-1}\right)}^{k-1} \mid X_{k-s}^{k-1}=x_{k-s}^{k-1}\right)\right] } \\
& =N_{n-\tau\left(x_{0}^{k-1}\right)}\left(x_{0}^{k-1}\right) \mathbb{P}\left(X_{k}^{k+\tau\left(x_{0}^{k-1}\right)-1}=x_{k-\tau\left(x_{0}^{k-1}\right)}^{k-1} \mid X_{k-s}^{k-1}=x_{k-s}^{k-1}\right) \\
& {\left[1-\mathbb{P}\left(X_{k}^{k+\tau\left(x_{0}^{k-1}\right)-1}=x_{k-\tau\left(x_{0}^{k-1}\right)}^{k-1} \mid X_{k-s}^{k-1}=x_{k-s}^{k-1}\right)\right] } \\
& \leq \frac{1}{4} N_{n-\tau\left(x_{0}^{k-1}\right)}\left(x_{0}^{k-1}\right) .
\end{aligned}
$$

Agora, fixe $\epsilon>0$. Considere

$$
f(\lambda)=\frac{\exp (\lambda)-1-\lambda}{\lambda}
$$

e

$$
g(\lambda)=\left(\frac{1}{2}+\epsilon\right) \lambda
$$

Cálculos diretos mostram que $f(\lambda)=g(\lambda)$ tem uma única solução, que chamamos $\lambda_{0}(\epsilon)$.

Para $0<\lambda<\lambda_{0}(\epsilon)$ temos $f(\lambda)<g(\lambda)$ e para $\lambda>\lambda_{0}(\epsilon)$ temos $f(\lambda)>g(\lambda)$. Desta forma, para $0<\lambda<\lambda_{0}(\epsilon)$

$$
\left\{X>\left(\frac{1}{2}+\epsilon\right) \lambda\right\} \subset\left\{X>\frac{\exp (\lambda)-1-\lambda}{\lambda}\right\} \text {. }
$$

Portanto, para todo $m \geq 0$ e $0<\lambda<\lambda_{0}(\epsilon)$, por (3.28) e (3.29)

$$
\left\{\theta^{m}<N_{n-\tau\left(x_{0}^{k-1}\right)}\left(x_{0}^{k-1}\right) \leq \theta^{m+1}\right\} \cap\left\{\left|Z_{n}\right|>\alpha+\left(\frac{1}{2}+\epsilon\right) \lambda \frac{\theta^{m+1}}{4}\right\} \subset\left\{\left|Z_{n}\right|>\alpha+\frac{\exp (\lambda)-1-\lambda}{\lambda} A_{n}\right\} .
$$

Iremos considerar $\alpha$ e $\lambda$ como

$$
\alpha(m)=\frac{\theta^{m / 2} \sqrt{\max [2 \xi k, \eta \log (m \log \theta)]}}{2(1+\epsilon)}
$$

e

$$
\lambda(m)=\frac{4 \sqrt{\max [2 \xi k, \eta \log (m \log \theta)]}}{(1+\epsilon) \theta^{1+m / 2}} .
$$

Para garantir que $\lambda<\lambda_{0}(\epsilon)$, necessário em (3.30), assumimos que $m$ satisfaz

$$
\theta^{m+1}>\tau(\epsilon) \max [2 \xi k, \eta \log (m \log \theta)] \quad \text { onde } \quad \tau(\epsilon)=\left(\frac{4}{\lambda_{0}(\epsilon)}\right)^{2}
$$

e também excede um patamar $m_{0}(\epsilon, \theta, \eta)$.

Considerando $\theta^{m}<N_{n-\tau\left(x_{0}^{k-1}\right)}\left(x_{0}^{k-1}\right)$, temos que

$$
\begin{aligned}
\alpha+\left(\frac{1}{2}+\epsilon\right) \lambda \frac{\theta^{m+1}}{4} & =\theta^{m / 2} \sqrt{\max [2 \xi k, \eta \log (m \log \theta)]} \\
& <\sqrt{N_{n-\tau\left(x_{0}^{k-1}\right)}\left(x_{0}^{k-1}\right) \max \left[2 \xi k, \eta \log \log \left(N_{n-\tau\left(x_{0}^{k-1}\right)}\left(x_{0}^{k-1}\right)\right)\right]}
\end{aligned}
$$

Além disso,

$$
\alpha \lambda=\mu \max [2 \xi k, \eta \log (m \log \theta)]
$$


onde

$$
\mu=\frac{2}{(1+\epsilon)^{2} \theta} .
$$

Assim, por (3.30), (3.31) e pelo Corolário A.10,

$$
\begin{aligned}
& \mathbb{P}\left(\bigcup_{n=k+\tau\left(x_{0}^{k-1}\right)}^{\infty}\left(\theta^{m}<N_{n-\tau\left(x_{0}^{k-1}\right)}\left(x_{0}^{k-1}\right) \leq \theta^{m+1}\right) \cap\left(\left|Z_{n}\right|>\sqrt{\left.N_{n-\tau\left(x_{0}^{k-1}\right)}\left(x_{0}^{k-1}\right) \max \left[2 \xi k, \eta \log \log N_{n-\tau\left(x_{0}^{k-1}\right)}\left(x_{0}^{k-1}\right)\right]\right)}\right)\right. \\
& <\mathbb{P}\left(\bigcup_{n=k+\tau\left(x_{0}^{k-1}\right)}^{\infty}\left(\theta^{m}<N_{n-\tau\left(x_{0}^{k-1}\right)}\left(x_{0}^{k-1}\right) \leq \theta^{m+1}\right) \cap\left(\left|Z_{n}\right|>\alpha+\left(\frac{1}{2}+\epsilon\right) \lambda \frac{\theta^{m+1}}{4}\right)\right) \\
& <\mathbb{P}\left(\bigcup_{n=k+\tau\left(x_{0}^{k-1}\right)}^{\infty}\left(\left|Z_{n}\right|>\alpha+\frac{\exp (\lambda)-1-\lambda}{\lambda} A_{n}\right)\right) \leq 2 \exp (-\lambda \alpha),
\end{aligned}
$$

onde $2 \exp (-\lambda \alpha)=2 \exp (-\mu \max [2 \xi k, \eta \log (m \log \theta)])$.

Note que a desigualdade (3.32) é válida quando $0<\lambda<\lambda_{0}(\epsilon)$, isto é, quando $m$ satisfaz $\theta^{m+1}>$ $\tau(\epsilon) \max [2 \xi k, \eta \log (m \log \theta)]$, com $\tau(\epsilon)=\left(\frac{4}{\lambda_{0}(\epsilon)}\right)^{2}$ e quando $m>m_{0}(\epsilon, \theta, \eta)$.

Da equação (3.27), para $n \geq k+\tau\left(x_{0}^{k-1}\right)$, observamos que

$$
\begin{aligned}
\frac{Z_{n}}{N_{n-\tau\left(x_{0}^{k-1}\right)}\left(x_{0}^{k-1}\right)} & =\frac{N_{n}\left(x_{0}^{k-1} x_{k-\tau\left(x_{0}^{k-1}\right)}^{k-1}\right)}{N_{n-\tau\left(x_{0}^{k-1}\right)}\left(x_{0}^{k-1}\right)}-\frac{\mathbb{P}\left(X_{k}^{k+\tau\left(x_{0}^{k-1}\right)-1}=x_{k-\tau\left(x_{0}^{k-1}\right)}^{k-1} \mid X_{k-s}^{k-1}=x_{k-s}^{k-1}\right) N_{n-\tau\left(x_{0}^{k-1}\right)}\left(x_{0}^{k-1}\right)}{N_{n-\tau\left(x_{0}^{k-1}\right)}\left(x_{0}^{k-1}\right)} \\
& =\hat{\rho}_{n}\left(x_{0}^{k-1}\right)-\mathbb{P}\left(X_{k}^{k+\tau\left(x_{0}^{k-1}\right)-1}=x_{k-\tau\left(x_{0}^{k-1}\right)}^{k-1} \mid X_{k-s}^{k-1}=x_{k-s}^{k-1}\right)
\end{aligned}
$$

e temos que $C_{m}\left(\xi, \eta, c, \theta, x_{0}^{k-1}\right)$ é igual a intersecção do evento

$\left\{\bigcup_{n=k+\tau\left(x_{0}^{k-1}\right)}^{\infty}\left(\theta^{m}<N_{n-\tau\left(x_{0}^{k-1}\right)}\left(x_{0}^{k-1}\right) \leq \theta^{m+1}\right) \cap\left(\left|Z_{n}\right|>\sqrt{\left.N_{n-\tau\left(x_{0}^{k-1}\right)}\left(x_{0}^{k-1}\right) \max \left[2 \xi k, \eta \log \log N_{n-\tau\left(x_{0}^{k-1}\right)}\left(x_{0}^{k-1}\right)\right]\right)}\right\}\right.$

com o evento

$$
\left\{N_{n-\tau\left(x_{0}^{k-1}\right)}\left(x_{0}^{k-1}\right)>2 c k\right\} .
$$

Quando a intersecção é não vazia, temos que $\theta^{m+1}>\tau(\epsilon) \max [2 \xi k, \eta \log (m \log \theta)]$ e como $\theta^{m+1} \geq$ $N_{n-\tau\left(x_{0}^{k-1}\right)}\left(x_{0}^{k-1}\right)>2 c k$, temos que $2 c k \geq \tau(\epsilon) \max [2 \xi k, \eta \log (m \log \theta)]$.

Assim, se $c$ em (3.22) é suficientemente grande, então para todo $m>m_{0}(\epsilon, \theta, \eta)$ e para todo $x_{0}^{k-1}$ com $k>s:$

$$
\begin{aligned}
\mathbb{P}\left(C_{m}\left(\xi, \eta, c, \theta, x_{0}^{k-1}\right)\right) \leq & 2 \exp (-\mu \max [2 \xi k, \eta \log (m \log \theta)]) \\
& = \begin{cases}2 \exp (-2 \mu \xi k), & \text { se } \quad k \geq \frac{\eta}{2 \xi} \log (m \log \theta) \\
2(m \log \theta)^{-\mu \eta}, & \text { se } k<\frac{\eta}{2 \xi} \log (m \log \theta) .\end{cases}
\end{aligned}
$$

Assuma $\theta$ e $\epsilon$ até agora arbitrários são selecionados tais que $\mu \xi=\frac{2 \xi}{(1+\epsilon)^{2} \theta}>\log |A|$, que é possível pela suposição $\xi>\frac{\log |A|}{2}$. De (3.7), temos que

$$
\mathbb{P}\left(C_{m}\right)=\mathbb{P}\left(\bigcup_{k \geq s} \bigcup_{x_{0}^{k-1}} C_{m}\left(\xi, \eta, c, \theta, x_{0}^{k-1}\right)\right) \leq \sum_{k \geq s} \sum_{x_{0}^{k-1}} \mathbb{P}\left(C_{m}\left(x_{0}^{k-1}\right)\right) .
$$


Utilizando a desigualdade (3.33),

$$
\begin{aligned}
\sum_{k \geq s} \sum_{x_{0}^{k-1}} \mathbb{P}\left(C_{m}\left(\xi, \eta, c, \theta, x_{0}^{k-1}\right)\right) & \leq 2\left[\sum_{k \geq \frac{\eta}{2 \xi} \log (m \log \theta)} \exp (-2 \mu \xi k)\left\{\# x_{0}^{k-1}: k \geq \frac{\eta}{2 \xi} \log (m \log \theta)\right\}\right] \\
& +2\left[\sum_{k<\frac{\eta}{2 \xi} \log (m \log \theta)}(m \log \theta)^{-\mu \eta}\left\{\# x_{0}^{k-1}: k<\frac{\eta}{2 \xi} \log (m \log \theta)\right\}\right] \\
& =2\left[\sum_{k \geq \frac{\eta}{2 \xi} \log (m \log \theta)}|A|^{k} \exp (-2 \mu \xi k)+\left(\sum_{k<\frac{\eta}{2 \xi} \log (m \log \theta)}|A|^{k}\right)(m \log \theta)^{-\mu \eta}\right] .
\end{aligned}
$$

Para o cálculo do primeiro termo do lado direito de (3.34), observe que

$$
\begin{aligned}
\sum_{k \geq \frac{\eta}{2 \xi} \log (m \log \theta)}^{\infty}|A|^{k} \exp (-2 \mu \xi k) & =\sum_{k \geq \frac{\eta}{2 \xi} \log (m \log \theta)}^{\infty} \exp (-k(2 \mu \xi-\log |A|)) \\
& =\frac{\exp \left(-\frac{\eta}{2 \xi} \log (m \log \theta)(2 \mu \xi-\log |A|)\right)}{1-\exp (-(2 \mu \xi-\log |A|))} .
\end{aligned}
$$

Para o segundo termo do lado direito da equação (3.34), temos que

$$
\left(\sum_{k<\frac{\eta}{2 \xi} \log (m \log \theta)}|A|^{k}\right)(m \log \theta)^{-\mu \eta} \leq(m \log \theta)^{-\mu \eta}|A|^{\frac{\eta}{2 \xi} \log (m \log \theta)+1} .
$$

Como

$$
|A|^{\frac{\eta}{2 \xi} \log (m \log \theta)}=(m \log \theta)^{\frac{\eta}{2 \xi} \log |A|},
$$

então temos por (3.35), (3.36) e (3.37),

$$
\begin{aligned}
\mathbb{P}\left(C_{m}\right) & \leq 2\left(\frac{\exp \left(-\frac{\eta}{2 \xi} \log (m \log \theta)(2 \mu \xi-\log |A|)\right)}{1-\exp (-(2 \mu \xi-\log |A|))}+(m \log \theta)^{-\mu \eta}|A|(m \log \theta)^{\frac{\eta}{2 \xi} \log |A|}\right) \\
& =2\left(\frac{(m \log \theta)^{-\eta \mu+\frac{\eta \log |A|}{2 \xi}}}{1-\exp (-(2 \mu \xi-\log |A|))}+|A|(m \log \theta)^{-\eta \mu+\frac{\eta \log |A|}{2 \xi}}\right) \\
& =\pi \times(m \log \theta)^{-\left(\mu-\frac{\log |A|}{2 \xi}\right) \eta},
\end{aligned}
$$

onde $\pi$ é constante.

Portanto, se $\eta>\frac{2 \xi}{2 \mu \xi-\log |A|}$, temos que $\sum_{m=1}^{\infty} \mathbb{P}\left(C_{m}\right)<\infty$, ou seja, $\mathbb{P}\left(\limsup C_{m}\right)=0$.

\subsubsection{Convergência do estimador $\hat{\rho}_{n}\left(x_{0}^{k-1}\right)$}

A seguir, verificaremos que, para uma sequência fixa $x_{0}^{k-1}$, pelo Lema A.11 e como consequência do Teorema 3.11, a distância entre a probabilidade condicional $\mathbb{P}\left(X_{k}^{\tau\left(x_{0}^{k-1}\right)+k-1}=x_{k-\tau\left(x_{0}^{k-1}\right)}^{k-1} \mid X_{k-s}^{k-1}=x_{k-s}^{k-1}\right)$ e seu estimador $\hat{\rho}_{n}\left(x_{0}^{k-1}\right)$ converge para zero.

Corolário 3.12. Sejam os conjuntos $G_{n}\left(\xi, \eta, x_{0}^{k-1}\right)$ e $H_{n}\left(c, x_{0}^{k-1}\right)$ definidos em (3.21) e (3.22). Sob as hipóteses do Teorema 3.11, temos que

$$
\mathbb{P}\left(\left\{G_{n}\left(\xi, \eta, x_{0}^{k-1}\right)\right\} i . v .\right)=0 .
$$

Prova. Considere $G_{n}\left(\xi, \eta, x_{0}^{k-1}\right)$ e $H_{n}\left(c, x_{0}^{k-1}\right)$ definidos em (3.21) e (3.22), respectivamente. Pelo 
Teorema 3.11 obtemos o seguinte resultado

$$
\mathbb{P}\left(\left\{G_{n}\left(\xi, \eta, x_{0}^{k-1}\right) \cap H_{n}\left(c, x_{0}^{k-1}\right)\right\} i . v .\right)=0 .
$$

Observe também que $H_{n}\left(c, x_{0}^{k-1}\right)$ é um conjunto crescente e existe um número inteiro positivo $n_{1}$ tal que

$$
N_{n-\tau\left(x_{0}^{k-1}\right)}\left(x_{0}^{k-1}\right)>2 c k,
$$

para todo $n>n_{1}$. Logo,

$$
\mathbb{P}\left(\left\{H_{n}\left(c, x_{0}^{k-1}\right)\right\} i . v .\right)=1
$$

Assim, pelo Lema A.11 podemos concluir, sob as condições do Teorema 3.11, que

$$
\mathbb{P}\left(\left\{G_{n}\left(\xi, \eta, x_{0}^{k-1}\right)\right\} i . v .\right)=0 .
$$

Logo,

$$
\mathbb{P}\left(\left\{\left|\hat{\rho}_{n}\left(x_{0}^{k-1}\right)-\mathbb{P}\left(X_{k}^{k+\tau\left(x_{0}^{k-1}\right)-1}=x_{k-\tau\left(x_{0}^{k-1}\right)}^{k-1} \mid X_{k-s}^{k-1}=x_{k-s}^{k-1}\right)\right|>\epsilon\left(\xi, \eta, x_{0}^{k-1}\right)\right\} i . v .\right)=0,
$$

e,

$$
\hat{\rho}_{n}\left(x_{0}^{k-1}\right) \underset{n \rightarrow \infty}{\stackrel{\text { q.c. }}{\longrightarrow}} \mathbb{P}\left(X_{k}^{k+\tau\left(x_{0}^{k-1}\right)-1}=x_{k-\tau\left(x_{0}^{k-1}\right)}^{k-1} \mid X_{k-s}^{k-1}=x_{k-s}^{k-1}\right) .
$$




\section{Apêndice A}

\section{Ferramentas auxiliares}

Nesta seção apresentaremos alguns lemas, definições e proposições necessários durante a prova dos teoremas 3.8 e 3.11 e dos corolários 3.9 e 3.12 .

O lema a seguir, utilizado na demonstração do Teorema 3.8, tem como objetivo mostrar que, sob determinadas condições, a sequência $\left(Z_{m}\right)_{m \in \mathbb{N}}$ definida em (A.1) é um martingal e $\left|Z_{n+1}-Z_{n}\right| \leq 1$.

Lema A.1. Seja $\left(X_{m}\right)_{m \in \mathbb{N}}$ um processo estocástico estacionário. Para $k>s$ e $x_{0}^{k-1}$ fixos, considere

$$
Z_{n}= \begin{cases}N_{n}\left(x_{0}^{k-1} x_{0}^{k-1}\right)-\mathbb{P}\left(X_{k}^{2 k-1}=x_{0}^{k-1} \mid X_{k-s}^{k-1}=x_{k-s}^{k-1}\right) N_{n-k}\left(x_{0}^{k-1}\right), & \text { para } n \geq 2 k \\ 0, & \text { para } 0 \leq n<2 k .\end{cases}
$$

Então, para todo $n \geq 2 k$,

(a) $Z_{n}$ é martingal;

(b) $\left|Z_{n+1}-Z_{n}\right| \leq 1$.

Prova. (a) $Z_{n}$ é martingal:

Considere $N_{n}(y)$ como definido em (3.1). A esperança condicional de $N_{n}\left(x_{0}^{k-1} x_{0}^{k-1}\right)$ dado $N_{n-1}\left(x_{0}^{k-1} x_{0}^{k-1}\right)$ e a esperança condicional de $N_{n-k}\left(x_{0}^{k-1}\right)$ dado $N_{n-k-1}\left(x_{0}^{k-1}\right)$ são dadas por

$$
\begin{aligned}
E\left(N_{n}\left(x_{0}^{k-1} x_{0}^{k-1}\right) \mid \mathcal{F}_{n-1}\right) & =E\left(N_{n}\left(x_{0}^{k-1} x_{0}^{k-1}\right) \mid N_{n-1}\left(x_{0}^{k-1} x_{0}^{k-1}\right)\right) \\
& =\left[N_{n-1}\left(x_{0}^{k-1} x_{0}^{k-1}\right)+1\right] \mathbb{P}\left(Y_{n-2 k}\left(x_{0}^{k-1} x_{0}^{k-1}\right)=1\right) \\
& +N_{n-1}\left(x_{0}^{k-1} x_{0}^{k-1}\right)\left[1-\mathbb{P}\left(Y_{n-2 k}\left(x_{0}^{k-1} x_{0}^{k-1}\right)=1\right)\right] \\
& =\mathbb{P}\left(Y_{n-2 k}\left(x_{0}^{k-1} x_{0}^{k-1}\right)=1\right)+N_{n-1}\left(x_{0}^{k-1} x_{0}^{k-1}\right) .
\end{aligned}
$$

e

$$
\begin{aligned}
E\left(N_{n-k}\left(x_{0}^{k-1}\right) \mid \mathcal{F}_{n-k-1}\right) & =E\left(N_{n-k}\left(x_{0}^{k-1}\right) \mid N_{n-k-1}\left(x_{0}^{k-1}\right)\right) \\
& =\left[N_{n-k-1}\left(x_{0}^{k-1}\right)+1\right] \mathbb{P}\left(Y_{n-2 k}\left(x_{0}^{k-1}\right)=1\right) \\
& +N_{n-k-1}\left(x_{0}^{k-1}\right)\left[1-\mathbb{P}\left(Y_{n-2 k}\left(x_{0}^{k-1}\right)=1\right)\right] \\
& =\mathbb{P}\left(Y_{n-2 k}\left(x_{0}^{k-1}\right)=1\right)+N_{n-k-1}\left(x_{0}^{k-1}\right) .
\end{aligned}
$$

Desta forma, considerando os resultados (A.2) e (A.3) na definição (A.1), temos

$$
\begin{aligned}
E\left(Z_{n} \mid Z_{n-1}\right) & =\mathbb{P}\left(Y_{n-2 k}\left(x_{0}^{k-1} x_{0}^{k-1}\right)=1\right)+N_{n-1}\left(x_{0}^{k-1} x_{0}^{k-1}\right) \\
& -\mathbb{P}\left(X_{k}^{2 k-1}=x_{0}^{k-1} \mid X_{k-s}^{k-1}=x_{k-s}^{k-1}\right) \mathbb{P}\left(Y_{n-2 k}\left(x_{0}^{k-1}\right)=1\right) \\
& -\mathbb{P}\left(X_{k}^{2 k-1}=x_{0}^{k-1} \mid X_{k-s}^{k-1}=x_{k-s}^{k-1}\right) N_{n-k-1}\left(x_{0}^{k-1}\right) .
\end{aligned}
$$


Como $\left(X_{m}\right)_{m \in \mathbb{N}}$ é estacionário,

$$
\begin{aligned}
E\left(Z_{n} \mid Z_{n-1}\right) & =\mathbb{P}\left(Y_{n-2 k}\left(x_{0}^{k-1} x_{0}^{k-1}\right)=1\right)+N_{n-1}\left(x_{0}^{k-1} x_{0}^{k-1}\right) \\
& -\mathbb{P}\left(X_{n-k}^{n-1}=x_{0}^{k-1} \mid X_{n-k-s}^{n-k-1}=x_{k-s}^{k-1}\right) \mathbb{P}\left(Y_{n-2 k}\left(x_{0}^{k-1}\right)=1\right) \\
& -\mathbb{P}\left(X_{k}^{2 k-1}=x_{0}^{k-1} \mid X_{k-s}^{k-1}=x_{k-s}^{k-1}\right) N_{n-k-1}\left(x_{0}^{k-1}\right) .
\end{aligned}
$$

Como

$$
\mathbb{P}\left(X_{n-k}^{n-1}=x_{0}^{k-1} \mid X_{n-k-s}^{n-k-1}=x_{k-s}^{k-1}\right) \mathbb{P}\left(Y_{n-2 k}\left(x_{0}^{k-1}\right)=1\right)=\mathbb{P}\left(X_{n-2 k}^{n-1}=x_{0}^{k-1} x_{0}^{k-1}\right),
$$

temos que

$$
\begin{aligned}
E\left(Z_{n} \mid Z_{n-1}\right) & =\mathbb{P}\left(X_{n-2 k}^{n-1}=x_{0}^{k-1} x_{0}^{k-1}\right)+N_{n-1}\left(x_{0}^{k-1} x_{0}^{k-1}\right) \\
& -\mathbb{P}\left(X_{n-2 k}^{n-1}=x_{0}^{k-1} x_{0}^{k-1}\right)-\mathbb{P}\left(X_{k}^{2 k-1}=x_{0}^{k-1} \mid X_{k-s}^{k-1}=x_{k-s}^{k-1}\right) N_{n-k-1}\left(x_{0}^{k-1}\right) \\
& =N_{n-1}\left(x_{0}^{k-1} x_{0}^{k-1}\right)-\mathbb{P}\left(X_{k}^{2 k-1}=x_{0}^{k-1} \mid X_{k-s}^{k-1}=x_{k-s}^{k-1}\right) N_{n-k-1}\left(x_{0}^{k-1}\right)=Z_{n-1} .
\end{aligned}
$$

(b) $\left|Z_{n+1}-Z_{n}\right| \leq 1$ :

$$
\begin{aligned}
\left|Z_{n+1}-Z_{n}\right| & =\mid N_{n+1}\left(x_{0}^{k-1} x_{0}^{k-1}\right)-N_{n}\left(x_{0}^{k-1} x_{0}^{k-1}\right) \\
& -\mathbb{P}\left(X_{k}^{2 k-1}=x_{0}^{k-1} \mid X_{k-s}^{k-1}=x_{k-s}^{k-1}\right)\left[N_{n-k+1}\left(x_{0}^{k-1}\right)-N_{n-k}\left(x_{0}^{k-1}\right)\right] \mid .
\end{aligned}
$$

Como

$$
\begin{aligned}
& 0 \leq N_{n+1}\left(x_{0}^{k-1} x_{0}^{k-1}\right)-N_{n}\left(x_{0}^{k-1} x_{0}^{k-1}\right) \leq 1 \quad \mathrm{e} \\
& 0 \leq N_{n-k+1}\left(x_{0}^{k-1}\right)-N_{n-k}\left(x_{0}^{k-1}\right) \leq 1
\end{aligned}
$$

Então podemos concluir que $\left|Z_{n+1}-Z_{n}\right|<1$.

O lema a seguir, utilizado na demonstração do Teorema 3.11, tem como objetivo mostrar que, sob determinadas condições, a sequência $\left(Z_{m}\right)_{m \in \mathbb{N}}$ definida em (A.4) é um martingal e $\left|Z_{n+1}-Z_{n}\right| \leq 1$.

Lema A.2. Seja $\left(X_{m}\right)_{m \in \mathbb{N}}$ um processo estocástico estacionário. Para $k>s$ e $x_{0}^{k-1}$ fixos, considere

$$
Z_{n}=\left\{\begin{array}{l}
N_{n}\left(x_{0}^{k-1} x_{k-\tau\left(x_{0}^{k-1}\right)}^{k-1}\right)-\mathbb{P}\left(X_{k}^{k+\tau\left(x_{0}^{k-1}\right)-1}=x_{k-\tau\left(x_{0}^{k-1}\right)}^{k-1} \mid X_{k-s}^{k-1}=x_{k-s}^{k-1}\right) N_{n-\tau\left(x_{0}^{k-1}\right)}\left(x_{0}^{k-1}\right), \quad \text { para } n \geq k+\tau\left(x_{0}^{k-1}\right) \\
0, \text { para } 0 \leq n<k+\tau\left(x_{0}^{k-1}\right) .
\end{array}\right.
$$

Então, para todo $n \geq k+\tau\left(x_{0}^{k-1}\right)$,

(a) $Z_{n}$ é martingal;

(b) $\left|Z_{n+1}-Z_{n}\right| \leq 1$.

Prova. (a) $Z_{n}$ é martingal:

Considere $N_{n}(y)$ como definido em (3.1). A esperança condicional de $N_{n}\left(x_{0}^{k-1} x_{k-\tau\left(x_{0}^{k-1}\right)}^{k-1}\right)$ dado $N_{n-1}\left(x_{0}^{k-1} x_{k-\tau\left(x_{0}^{k-1}\right)}^{k-1}\right)$ e a esperança condicional de $N_{n-\tau\left(x_{0}^{k-1}\right)}\left(x_{0}^{k-1}\right)$ dado $N_{n-\tau\left(x_{0}^{k-1}\right)-1}\left(x_{0}^{k-1}\right)$ 
são dadas por

$$
\begin{aligned}
& E\left(N_{n}\left(x_{0}^{k-1} x_{k-\tau\left(x_{0}^{k-1}\right)}^{k-1}\right) \mid \mathcal{F}_{n-1}\right)=E\left(N_{n}\left(x_{0}^{k-1} x_{k-\tau\left(x_{0}^{k-1}\right)}^{k-1}\right) \mid N_{n-1}\left(x_{0}^{k-1} x_{k-\tau\left(x_{0}^{k-1}\right)}^{k-1}\right)\right) \\
& =\left[N_{n-1}\left(x_{0}^{k-1} x_{k-\tau\left(x_{0}^{k-1}\right)}^{k-1}\right)+1\right] \mathbb{P}\left(Y_{n-\tau\left(x_{0}^{k-1}\right)-k}\left(x_{0}^{k-1} x_{k-\tau\left(x_{0}^{k-1}\right)}^{k-1}\right)=1\right) \\
& +N_{n-1}\left(x_{0}^{k-1} x_{k-\tau\left(x_{0}^{k-1}\right)}^{k-1}\right)\left[1-\mathbb{P}\left(Y_{n-\tau\left(x_{0}^{k-1}\right)-k}\left(x_{0}^{k-1} x_{k-\tau\left(x_{0}^{k-1}\right)}^{k-1}\right)=1\right)\right] \\
& =\mathbb{P}\left(Y_{n-\tau\left(x_{0}^{k-1}\right)-k}\left(x_{0}^{k-1} x_{k-\tau\left(x_{0}^{k-1}\right)}^{k-1}\right)=1\right)+N_{n-1}\left(x_{0}^{k-1} x_{k-\tau\left(x_{0}^{k-1}\right)}^{k-1}\right)
\end{aligned}
$$

e

$$
\begin{aligned}
E\left(N_{n-\tau\left(x_{0}^{k-1}\right)}\left(x_{0}^{k-1}\right) \mid \mathcal{F}_{n-\tau\left(x_{0}^{k-1}\right)-1}\right) & =E\left(N_{n-\tau\left(x_{0}^{k-1}\right)}\left(x_{0}^{k-1}\right) \mid N_{n-\tau\left(x_{0}^{k-1}\right)-1}\left(x_{0}^{k-1}\right)\right) \\
& =\left[N_{n-\tau\left(x_{0}^{k-1}\right)-1}\left(x_{0}^{k-1}\right)+1\right] \mathbb{P}\left(Y_{n-k-\tau\left(x_{0}^{k-1}\right)}\left(x_{0}^{k-1}\right)=1\right) \\
& +N_{n-\tau\left(x_{0}^{k-1}\right)-1}\left(x_{0}^{k-1}\right)\left[1-\mathbb{P}\left(Y_{n-k-\tau\left(x_{0}^{k-1}\right)}\left(x_{0}^{k-1}\right)=1\right)\right] \\
& =\mathbb{P}\left(Y_{n-k-\tau\left(x_{0}^{k-1}\right)}\left(x_{0}^{k-1}\right)=1\right)+N_{n-\tau\left(x_{0}^{k-1}\right)-1}\left(x_{0}^{k-1}\right) .
\end{aligned}
$$

Desta forma, considerando os resultados (A.5) e (A.6) na definição (A.4), temos

$$
\begin{aligned}
E\left(Z_{n} \mid Z_{n-1}\right) & =\mathbb{P}\left(Y_{n-k-\tau\left(x_{0}^{k-1}\right)}\left(x_{0}^{k-1} x_{k-\tau\left(x_{0}^{k-1}\right)}^{k-1}\right)=1\right)+N_{n-1}\left(x_{0}^{k-1} x_{k-\tau\left(x_{0}^{k-1}\right)}^{k-1}\right) \\
& -\mathbb{P}\left(X_{k}^{k+\tau\left(x_{0}^{k-1}\right)-1}=x_{k-\tau\left(x_{0}^{k-1}\right)}^{k-1} \mid X_{k-s}^{k-1}=x_{k-s}^{k-1}\right) \mathbb{P}\left(Y_{n-k-\tau\left(x_{0}^{k-1}\right)}\left(x_{0}^{k-1}\right)=1\right) \\
& -\mathbb{P}\left(X_{k}^{k+\tau\left(x_{0}^{k-1}\right)-1}=x_{k-\tau\left(x_{0}^{k-1}\right)}^{k-1} \mid X_{k-s}^{k-1}=x_{k-s}^{k-1}\right) N_{n-\tau\left(x_{0}^{k-1}\right)-1}\left(x_{0}^{k-1}\right) .
\end{aligned}
$$

Como $\left(X_{m}\right)_{m \in \mathbb{N}}$ é estacionário,

$$
\begin{aligned}
E\left(Z_{n} \mid Z_{n-1}\right) & =\mathbb{P}\left(Y_{n-k-\tau\left(x_{0}^{k-1}\right)}\left(x_{0}^{k-1} x_{k-\tau\left(x_{0}^{k-1}\right)}^{k-1}\right)=1\right)+N_{n-1}\left(x_{0}^{k-1} x_{k-\tau\left(x_{0}^{k-1}\right)}^{k-1}\right) \\
& -\mathbb{P}\left(X_{n-\tau\left(x_{0}^{k-1}\right)}^{n-1}=x_{k-\tau\left(x_{0}^{k-1}\right)}^{k-1} \mid X_{n-\tau\left(x_{0}^{k-1}\right)-s}^{n-\tau\left(x^{k-1}\right)-1}=x_{k-s}^{k-1}\right) \mathbb{P}\left(Y_{n-k-\tau\left(x_{0}^{k-1}\right)}\left(x_{0}^{k-1}\right)=1\right) \\
& -\mathbb{P}\left(X_{k}^{k+\tau\left(x_{0}^{k-1}\right)-1}=x_{k-\tau\left(x_{0}^{k-1}\right)}^{k-1} \mid X_{k-s}^{k-1}=x_{k-s}^{k-1}\right) N_{n-\tau\left(x_{0}^{k-1}\right)-1}\left(x_{0}^{k-1}\right) .
\end{aligned}
$$

Como

$$
\begin{aligned}
& \mathbb{P}\left(X_{n-\tau\left(x_{0}^{k-1}\right)}^{n-1}=x_{k-\tau\left(x_{0}^{k-1}\right)}^{k-1} \mid X_{n-\tau\left(x_{0}^{k-1}\right)-s}^{n-\tau\left(x_{k}^{k-1}\right)-1}=x_{k-s}^{k-1}\right) \mathbb{P}\left(Y_{n-k-\tau\left(x_{0}^{k-1}\right)}\left(x_{0}^{k-1}\right)=1\right) \\
& =\mathbb{P}\left(X_{n-k-\tau\left(x_{0}^{k-1}\right)}^{n-1}=x_{0}^{k-1} x_{k-\tau\left(x_{0}^{k-1}\right)}^{k-1}\right)
\end{aligned}
$$


temos que

$$
\begin{aligned}
& E\left(Z_{n} \mid Z_{n-1}\right)=\mathbb{P}\left(X_{n-k-\tau\left(x_{0}^{k-1}\right)}^{n-1}=x_{0}^{k-1} x_{k-\tau\left(x_{0}^{k-1}\right)}^{k-1}\right)+N_{n-1}\left(x_{0}^{k-1} x_{k-\tau\left(x_{0}^{k-1}\right)}^{k-1}\right) \\
& -\mathbb{P}\left(X_{n-\tau\left(x_{0}^{k-1}\right)-k}^{n-1}=x_{0}^{k-1} x_{k-\tau\left(x_{0}^{k-1}\right)}^{k-1}\right) \\
& -\mathbb{P}\left(X_{k}^{k+\tau\left(x_{0}^{k-1}\right)-1}=x_{k-\tau\left(x_{0}^{k-1}\right)}^{k-1} \mid X_{k-s}^{k-1}=x_{k-s}^{k-1}\right) N_{n-\tau\left(x_{0}^{k-1}\right)-1}\left(x_{0}^{k-1}\right) \\
& =N_{n-1}\left(x_{0}^{k-1} x_{k-\tau\left(x_{0}^{k-1}\right)}^{k-1}\right)-\mathbb{P}\left(X_{k}^{k+\tau\left(x_{0}^{k-1}\right)-1}=x_{k-\tau\left(x_{0}^{k-1}\right)}^{k-1} \mid X_{k-s}^{k-1}=x_{k-s}^{k-1}\right) N_{n-\tau\left(x_{0}^{k-1}\right)-1}\left(x_{0}^{k-1}\right) \\
& =Z_{n-1} .
\end{aligned}
$$

(b) $\left|Z_{n+1}-Z_{n}\right| \leq 1$ :

$$
\begin{aligned}
\left|Z_{n+1}-Z_{n}\right| & =\mid N_{n+1}\left(x_{0}^{k-1} x_{k-\tau\left(x_{0}^{k-1}\right)}^{k-1}\right)-N_{n}\left(x_{0}^{k-1} x_{k-\tau\left(x_{0}^{k-1}\right)}^{k-1}\right) \\
& -\mathbb{P}\left(X_{k}^{k+\tau\left(x_{0}^{k-1}\right)-1}=x_{k-\tau\left(x_{0}^{k-1}\right)}^{k-1} \mid X_{k-s}^{k-1}=x_{k-s}^{k-1}\right)\left[N_{n-\tau\left(x_{0}^{k-1}\right)+1}\left(x_{0}^{k-1}\right)-N_{n-\tau\left(x_{0}^{k-1}\right)}\left(x_{0}^{k-1}\right)\right] \mid .
\end{aligned}
$$

Como

$$
\begin{aligned}
& 0 \leq N_{n+1}\left(x_{0}^{k-1} x_{k-\tau\left(x_{0}^{k-1}\right)}^{k-1}\right)-N_{n}\left(x_{0}^{k-1} x_{k-\tau\left(x_{0}^{k-1}\right)}^{k-1}\right) \leq 1 \quad \mathrm{e} \\
& 0 \leq N_{n-\tau\left(x_{0}^{k-1}\right)+1}\left(x_{0}^{k-1}\right)-N_{n-\tau\left(x_{0}^{k-1}\right)}\left(x_{0}^{k-1}\right) \leq 1
\end{aligned}
$$

Então podemos concluir que $\left|Z_{n+1}-Z_{n}\right|<1$.

A seguir, apresentaremos as definições de martingal, martingal quadrado-integrável, submartingal e supermartingal. Estas definições basearam-se em ([10]).

Definição A.3. Seja $\left(Z_{m}\right)_{m \in \mathbb{N}}$ uma sequência de variáveis aleatórias integráveis sobre o espaço de probabilidade $(\Omega, \mathcal{F}, \mathbb{P})$ e $\mathcal{F}_{1} \subset \mathcal{F}_{2} \subset \cdots$ uma sequência crescente de sub- $\sigma$-álgebras de $\mathcal{F}$. Assumimos que $Z_{m}$ é $\mathcal{F}_{m}$-mensurável. Dizemos que a sequência $\left(Z_{m}\right)_{m \in \mathbb{N}}$ é um martingal relativo a $\mathcal{F}_{m}$ se e somente se, para todo $m \in \mathbb{N}$,

$$
E\left(Z_{m+1} \mid \mathcal{F}_{m}\right)=Z_{m} \quad \text { q.c. }
$$

Obs: Se a condição $E\left(Z_{m}^{2}\right)<\infty$ é satisfeita para todo $m \in \mathbb{N}$, dizemos que a sequência $\left(Z_{m}\right)_{m \in \mathbb{N}}$ é um martingal quadrado-integrável relativo a $\mathcal{F}_{m}$.

A sequência $\left(Z_{m}\right)_{m \in \mathbb{N}}$ é dita ser um submartingal relativo a $\mathcal{F}_{m}$ se e somente se, para todo $m \in \mathbb{N}$,

$$
E\left(Z_{m+1} \mid \mathcal{F}_{m}\right) \geq Z_{m} \quad q . c .
$$

Dizemos que a sequência $\left(Z_{m}\right)_{m \in \mathbb{N}}$ é um supermartingal relativo a $\mathcal{F}_{m}$ se e somente se, para todo $m \in \mathbb{N}$,

$$
E\left(Z_{m+1} \mid \mathcal{F}_{m}\right) \leq Z_{m} \quad q . c .
$$

O próximo lema nos mostra que o quadrado de uma sequência martingal relativo a $\mathcal{F}_{m}$ é uma sequência submartingal relativo a $\mathcal{F}_{m}$. 
Lema A.4. Para todo martingal $\left(Z_{m}\right)_{m \in \mathbb{N}}$ relativo a $\mathcal{F}_{m}$, a sequência $\left(Z_{m}^{2}\right)_{m \in \mathbb{N}}$ é um submartingal relativo a $\mathcal{F}_{m}$.

Prova. De acordo com a Desigualdade de Schwarz para esperança condicional, temos que

$$
E\left(Z_{n+1}^{2} \mid \mathcal{F}_{n}\right) \geq\left[E\left(Z_{n+1} \mid \mathcal{F}_{n}\right)\right]^{2}=Z_{n}^{2}
$$

para todo $n \in \mathbb{N}$.

A seguir apresentaremos a Decomposição de Doob para martingais, onde um submartingal relativo a $\mathcal{F}_{n}$ pode ser escrito como a soma de um martingal relativo a $\mathcal{F}_{n}$ e uma sequência crescente previsível relativa a $\mathcal{F}_{n-1}$, para todo $n \in \mathbb{N}$. Esta definição baseou-se em ([30]).

Definição A.5. Considere agora $\left(Z_{m}^{2}\right)_{m \in \mathbb{N}}$ um submartingal relativo a $\mathcal{F}_{m}$. Então existe um martingal $\left(M_{m}\right)_{m \in \mathbb{N}}$ relativo a $\mathcal{F}_{m}$ e uma sequência crescente previsível $\left(A_{m}\right)_{m \in \mathbb{N}}$ relativo a $\mathcal{F}_{m-1}$ tal que, para todo $n \in \mathbb{N}$, a Decomposição de Doob

$$
Z_{n}^{2}=M_{n}+A_{n} \quad q . c .
$$

é válida. Uma decomposição deste tipo é única.

Temos que $M_{0}=X_{0}, A_{0}=0 e$

$$
\begin{aligned}
& M_{n}=M_{0}+\sum_{j=0}^{n-1}\left[Z_{j+1}^{2}-E\left(Z_{j+1}^{2} \mid \mathcal{F}_{j}\right)\right], \\
& \left.A_{n}=\sum_{j=0}^{n-1}\left[E\left(Z_{j+1}^{2} \mid \mathcal{F}_{j}\right)-Z_{j}^{2}\right]=\sum_{j=0}^{n-1}\left[E\left(Z_{j+1}-Z_{j}\right)^{2} \mid \mathcal{F}_{j}\right)\right] .
\end{aligned}
$$

Por definição,

$$
A_{n+1}-A_{n}=E\left(Z_{n+1}^{2} \mid \mathcal{F}_{n}\right)-Z_{n}^{2}=E\left[\left(Z_{n+1}-Z_{n}\right)^{2} \mid \mathcal{F}_{n}\right]
$$

para todo $n \in \mathbb{N}$.

O lema a seguir, baseado em ([25]), nos mostra que, sob determinadas condições, a sequência exponencial dependente do martingal quadrado-integrável e da sequência crescente previsível, é um supermartingal positivo. Este lema será utilizado no Corolário A.10.

Lema A.6. Seja $\left(Z_{m}\right)_{m \in \mathbb{N}}$ um martingal quadrado integrável tal que $\sup \left(Z_{n+1}-Z_{n}\right) \leq 1$ q.c. Então, para todo $\lambda \in \mathbb{R}_{+}$, a sequência de variáveis aleatórias

$$
\exp \left(\lambda Z_{n}-\phi(\lambda) A_{n}\right)
$$

é um supermartingal positivo. Neste caso, $A_{n}$ é tal que $Z_{n}^{2}=M_{n}+A_{n}$ q.c.

Nota: Seja $\phi: \mathbb{R}_{+} \rightarrow \mathbb{R}_{+}$a função definida por $\phi(\lambda)=\exp (\lambda)-1-\lambda$. Então existe um $\delta>0$ tal que para todo $\lambda_{0} \in(-\delta, \delta)$, temos que $\phi\left(\lambda_{0}\right)=\frac{1}{2} \lambda_{0}^{2}+o\left(\lambda_{0}^{2}\right)$.

Prova. Inicialmente, mostraremos que a desigualdade

$$
\exp (\lambda y) \leq 1+\lambda y+\phi(\lambda) y^{2}
$$

é válida para todo número real $y \in]-\infty, 1]$. Para isto, consideraremos dois casos de acordo com o intervalo de $y$. 
Caso 1: $y \in]-\infty, 0]$

Como $y \leq 0$ e $\lambda>0$, temos que $\exp (\lambda y) \leq 1$. Integrando duas vezes a desigualdade $\exp (\lambda y) \leq 1$ no intervalo $[y, 0]$, obtemos

$$
-1-\lambda y+\exp (\lambda y) \leq \frac{1}{2} y^{2} \lambda^{2}
$$

Portanto, para $y \in]-\infty, 0]$,

$$
\exp (\lambda y) \leq 1+\lambda y+\frac{1}{2} \lambda^{2} y^{2} \leq 1+\lambda y+y^{2} \phi(\lambda)
$$

pois $\phi(\lambda) \geq \frac{1}{2} \lambda^{2}$

Caso 2: $y \in[0,1]$

Utilizando a expansão de séries da função $\exp (\lambda y)$ ao redor do ponto 0 , quando $y \in[0,1]$, temos

$$
\exp (\lambda y)=1+\lambda y+\sum_{n \geq 2} \frac{\lambda^{n} y^{2} y^{n-2}}{n !} \leq 1+\lambda y+y^{2} \sum_{n \geq 2} \frac{\lambda^{n} 1^{n-2}}{n !}=1+\lambda y+y^{2} \phi(\lambda) .
$$

Portanto, para $y \in[0,1]$,

$$
\exp (\lambda y) \leq 1+\lambda y+y^{2} \phi(\lambda)
$$

Logo, podemos concluir que para todo número real $y \in]-\infty, 1]$ temos que

$$
\exp (\lambda y) \leq 1+\lambda y+\phi(\lambda) y^{2} .
$$

A seguir, considerando a suposição $\sup \left(Z_{n+1}-Z_{n}\right) \leq 1$ q.c., por (A.7),

$$
\begin{aligned}
E\left(\exp \left(\lambda\left(Z_{n+1}-Z_{n}\right)\right) \mid \mathcal{F}_{n}\right) & \leq E\left(\left(1+\lambda\left(Z_{n+1}-Z_{n}\right)+\left(Z_{n+1}-Z_{n}\right)^{2} \phi(\lambda)\right) \mid \mathcal{F}_{n}\right) \\
& =1+\lambda E\left(Z_{n+1} \mid \mathcal{F}_{n}\right)-\lambda Z_{n}+\phi(\lambda) E\left(\left(Z_{n+1}-Z_{n}\right)^{2} \mid \mathcal{F}_{n}\right) .
\end{aligned}
$$

Como $\left(Z_{m}\right)_{m \in \mathbb{N}}$ é um martingal quadrado integrável, pela Decomposição de Doob, para todo $n \in \mathbb{N}$ vale que

$$
A_{n+1}-A_{n}=E\left(\left(Z_{n+1}-Z_{n}\right)^{2} \mid \mathcal{F}_{n}\right),
$$

onde $A_{n}$ é o processo crescente. Então,

$$
E\left(\exp \left(\lambda\left(Z_{n+1}-Z_{n}\right)\right) \mid \mathcal{F}_{n}\right) \leq 1+\phi(\lambda) E\left(\left(Z_{n+1}-Z_{n}\right)^{2} \mid \mathcal{F}_{n}\right)=1+\phi(\lambda)\left(A_{n+1}-A_{n}\right),
$$

para todo $n \in \mathbb{N}$. Como $\phi(\lambda)\left(A_{n+1}-A_{n}\right)>0$, então

$$
1+\phi(\lambda)\left(A_{n+1}-A_{n}\right) \leq \exp \left(\phi(\lambda)\left(A_{n+1}-A_{n}\right)\right) .
$$

Deste modo,

$$
E\left(\exp \left(\lambda\left(Z_{n+1}-Z_{n}\right)\right) \mid \mathcal{F}_{n}\right) \leq \exp \left(\phi(\lambda)\left(A_{n+1}-A_{n}\right)\right)
$$

ou seja,

$$
E\left(\exp \left(\lambda Z_{n+1}-\phi(\lambda) A_{n+1}\right) \mid \mathcal{F}_{n}\right) \leq \exp \left(\lambda Z_{n}-\phi(\lambda) A_{n}\right),
$$

para todo $n \in \mathbb{N}$.

A seguir, apresentaremos as definições de Tempo de Markov e Tempo de Parada. Para isto, utilizamos a referência ([30]). 
Definição A.7. Uma variável aleatória $\nu$ com valores no conjunto $\{0,1, \ldots\} \cup\{\infty\}$ é um Tempo de Markov com respeito a $\mathcal{F}_{n}$ ou, uma variável aleatória independente do futuro se, para todo $n \in \mathbb{N}$,

$$
\{\nu=n\} \in \mathcal{F}_{n} .
$$

Quando $\mathbb{P}(\nu<\infty)=1$, um Tempo de Markov $\nu$ é chamado Tempo de Parada.

O lema a seguir mostra que, sob determinadas condições a respeito do processo e do tempo de parada, a combinação de dois supermartingais também é um supermartingal. A referência utilizada foi ([25]) e este lema será utilizado na Proposição A.9.

Lema A.8. Seja $\left(Z_{m}^{(i)}\right)_{m \in \mathbb{N}}$, com $i=1,2$, dois supermartingais assumindo valores em $\mathbb{R}_{+}$e considere $\nu$ um tempo de parada tal que $Z_{\nu}^{(1)} \geq Z_{\nu}^{(2)}$ sobre $\{\nu<\infty\}$. Então

$$
Z_{n}(w)=\left\{\begin{array}{lll}
Z_{n}^{(1)}(w) & \text { se } & n<\nu(w) \\
Z_{n}^{(2)}(w) & \text { se } & n \geq \nu(w)
\end{array} \quad(n \in \mathbb{N})\right.
$$

define um supermartingal assumindo valores em $\mathbb{R}_{+}$.

Prova. Para todo $n \in \mathbb{N}$, temos que $Z_{n}$ é $\mathcal{F}_{n}$-mensurável. Observe que (A.8) pode ser escrito como

$$
Z_{n}=\mathbb{1}\{\nu>n\} Z_{n}^{(1)}+\mathbb{1}\{\nu \leq n\} Z_{n}^{(2)} .
$$

Temos que $\left(Z_{m}^{(1)}\right)_{m \in \mathbb{N}}$ e $\left(Z_{m}^{(2)}\right)_{m \in \mathbb{N}}$ são supermartingais assumindo valores em $\mathbb{R}_{+}$. Logo,

$$
\begin{aligned}
Z_{n} & \geq \mathbb{1}\{\nu>n\} E\left(Z_{n+1}^{(1)} \mid \mathcal{F}_{n}\right)+\mathbb{1}\{\nu \leq n\} E\left(Z_{n+1}^{(2)} \mid \mathcal{F}_{n}\right) \\
& =E\left[\mathbb{1}\{\nu>n\} Z_{n+1}^{(1)}+\mathbb{1}\{\nu \leq n\} Z_{n+1}^{(2)} \mid \mathcal{F}_{n}\right] .
\end{aligned}
$$

Pela suposição $Z_{\nu}^{(1)} \geq Z_{\nu}^{(2)}$ sobre $\{\nu<\infty\}$ temos que

$$
\begin{aligned}
\mathbb{1}\{\nu>n\} Z_{n+1}^{(1)}+\mathbb{1}\{\nu \leq n\} Z_{n+1}^{(2)} & =\mathbb{1}\{\nu \geq n+1\} Z_{n+1}^{(1)}+\mathbb{1}\{\nu \leq n\} Z_{n+1}^{(2)} \\
& \geq \mathbb{1}\{\nu>n+1\} Z_{n+1}^{(1)}+\mathbb{1}\{\nu \leq n+1\} Z_{n+1}^{(2)}=Z_{n+1} .
\end{aligned}
$$

Portanto, $Z_{n} \geq E\left(Z_{n+1} \mid \mathcal{F}_{n}\right)$.

A proposição a seguir, cuja referência foi ([25]), mostra que, para um supermartingal positivo $Z_{n}$, a variável aleatória $\sup _{n \in \mathbb{N}} Z_{n}$ é q.c. finita sobre o conjunto $\left\{Z_{0}<\infty\right\}$. Além disso, encontramos um limite superior para a probabilidade condicional desta variável ser maior que uma determinada constante. Este lema será utilizado no Corolário A.10.

Proposição A.9. Seja $\left(Z_{m}\right)_{m \in \mathbb{N}}$ um supermartingal positivo. Então, para toda constante $a>0, a$ variável aleatória

$$
\sup _{n \in \mathbb{N}} Z_{n}
$$

é q.c. finita sobre o conjunto $\left\{Z_{0}<\infty\right\}$, e satisfaz a seguinte desigualdade

$$
\mathbb{P}\left(\sup _{n \in \mathbb{N}} Z_{n}>a \mid \mathcal{F}_{0}\right) \leq \min \left(\frac{Z_{0}}{a}, 1\right) .
$$


Prova. Primeiramente, veremos que sobre o conjunto $\left\{Z_{0}<\infty\right\}$, a desigualdade (A.9) é satisfeita. Seja $\left(Z_{m}\right)_{m \in \mathbb{N}}$ um supermartingal positivo e $\nu_{a}$ o tempo de parada definido por

$$
\nu_{a}=\left\{\begin{array}{cl}
\min \left(n: Z_{n}>a\right) & \text { se } \sup _{n \in \mathbb{N}} Z_{n}>a \\
\infty & \text { se } \sup _{n \in \mathbb{N}} Z_{n} \leq a .
\end{array} \quad(a>0)\right.
$$

Como $Z_{\nu_{a}}>a$ sobre $\left\{\nu_{a}<\infty\right\}$ e como a constante $a$ é um supermartingal pois $E\left(a \mid \mathcal{F}_{n-1}\right) \leq a$, pelo Lema A.8 temos que

$$
Y_{n}=\left\{\begin{array}{lll}
Z_{n} & \text { se } & n<\nu_{a} \\
a & \text { se } & n \geq \nu_{a}
\end{array} \quad(n \in \mathbb{N})\right.
$$

é um supermartingal positivo. Assim, $Y_{0} \geq E\left(Y_{n} \mid \mathcal{F}_{0}\right)$.

Observe que no conjunto $\left\{Z_{0}<\infty\right\}, Y_{0}$ toma o valor $Z_{0}$ quando $Z_{0} \leq a$ ou $Y_{0}$ toma o valor $a$ quando $Z_{0}>a$. Desta forma,

$$
Y_{0}=\min \left\{Z_{0}, a\right\}
$$

Por (A.11), podemos escrever

$$
Y_{n}=Z_{n} \mathbb{1}\left\{\nu_{a}>n\right\}+a \mathbb{1}\left\{\nu_{a} \leq n\right\} \geq a \mathbb{1}\left\{\nu_{a} \leq n\right\},
$$

e temos que

$$
Y_{0}=\min \left(Z_{0}, a\right) \geq E\left(Y_{n} \mid \mathcal{F}_{0}\right) \geq E\left(a \mathbb{1}\left\{\nu_{a} \leq n\right\} \mid \mathcal{F}_{0}\right)=a \mathbb{P}\left(\nu_{a} \leq n \mid \mathcal{F}_{0}\right) .
$$

Então,

$$
a \mathbb{P}\left(\nu_{a} \leq n \mid \mathcal{F}_{0}\right) \leq \min \left(Z_{0}, a\right) .
$$

Dividindo (A.12) por $a$, obtemos

$$
\mathbb{P}\left(\nu_{a} \leq n \mid \mathcal{F}_{0}\right) \leq \min \left(\frac{Z_{0}}{a}, 1\right)
$$

A seguir veremos que, sobre o conjunto $\left\{Z_{0}<\infty\right\}$, a variável aleatória $\sup _{n \in \mathbb{N}} Z_{n}$ é q.c. finita. Considerando $A_{n}=\left\{\nu_{a} \leq n\right\}$, temos que $A_{n}$ são conjuntos crescentes. Desta forma,

$$
\bigcup_{n \in \mathbb{N}} A_{n}=\bigcup_{n \in \mathbb{N}}\left\{\nu_{a} \leq n\right\}=\left\{\nu_{a}<\infty\right\} .
$$

Pela definição (A.10), temos que $\left\{\nu_{a}<\infty\right\}=\left\{\sup _{n \in \mathbb{N}} Z_{n}>a\right\}$. Tomando $n \uparrow \infty$, por (A.13), temos que

$$
\mathbb{P}\left(\sup _{n \in \mathbb{N}} Z_{n}>a \mid \mathcal{F}_{0}\right)=\mathbb{P}\left(\nu_{a}<\infty \mid \mathcal{F}_{0}\right)=\lim _{n \rightarrow \infty} \mathbb{P}\left(\nu_{a} \leq n \mid \mathcal{F}_{0}\right) \leq \min \left(\frac{Z_{0}}{a}, 1\right)
$$

Integrando ambos os lados desta desigualdade sobre o evento $\left\{Z_{0}<\infty\right\}$ que pertence a $\mathcal{F}_{0}$, temos

$$
\int_{\left\{Z_{0}<\infty\right\}} \mathbb{P}\left(\sup _{n \in \mathbb{N}} Z_{n}>a \mid \mathcal{F}_{0}\right) d \mathbb{P} \leq \int_{\left\{Z_{0}<\infty\right\}} \min \left(\frac{Z_{0}}{a}, 1\right) d \mathbb{P} .
$$

Utilizando a igualdade

$$
\int_{B} \mathbb{P}(A \mid \mathcal{F}) d \mathbb{P}=\mathbb{P}(A B)
$$


válida quando $B \in \mathcal{F}$, o lado esquerdo da equação (A.14) é dado por

$$
\int_{\left\{Z_{0}<\infty\right\}} \mathbb{P}\left(\sup _{n \in \mathbb{N}} Z_{n}>a \mid \mathcal{F}_{0}\right) d \mathbb{P}=\mathbb{P}\left(Z_{0}<\infty, \sup _{n \in \mathbb{N}} Z_{n}>a\right) .
$$

Logo, obtemos

$$
\mathbb{P}\left(Z_{0}<\infty, \sup _{n \in \mathbb{N}} Z_{n}>a\right) \leq \int_{\left\{Z_{0}<\infty\right\}} \min \left(\frac{Z_{0}}{a}, 1\right) d \mathbb{P} .
$$

Tomando $a \uparrow \infty$ em ambos os lados da equação (A.15), temos

$$
\lim _{a \rightarrow \infty} \mathbb{P}\left(Z_{0}<\infty, \sup _{n \in \mathbb{N}} Z_{n}>a\right) \leq \lim _{a \rightarrow \infty} \int_{\left\{Z_{0}<\infty\right\}} \min \left(\frac{Z_{0}}{a}, 1\right) d \mathbb{P} .
$$

Considerando $C_{a}=\left\{\sup _{n \in \mathbb{N}} Z_{n}>a\right\}$, temos que $C_{a}$ são conjuntos decrescentes. Desta forma,

$$
\bigcap_{a \in \mathbb{R}} C_{a}=\bigcap_{a \in \mathbb{R}}\left\{\sup _{n \in \mathbb{N}} Z_{n}>a\right\}=\left\{\sup _{n \in \mathbb{N}} Z_{n}=\infty\right\}
$$

Logo, considerando o lado esquerdo da equação (A.16) temos

$$
\lim _{a \rightarrow \infty} \mathbb{P}\left(Z_{0}<\infty, \sup _{n \in \mathbb{N}} Z_{n}>a\right)=\mathbb{P}\left(Z_{0}<\infty, \sup _{n \in \mathbb{N}} Z_{n}=\infty\right)
$$

Considerando o lado direito da equação (A.16) temos

$$
\lim _{a \rightarrow \infty} \int_{\left\{Z_{0}<\infty\right\}} \min \left(\frac{Z_{0}}{a}, 1\right) d \mathbb{P}=0
$$

Portanto, por (A.17) e (A.18),

$$
\mathbb{P}\left(Z_{0}<\infty, \sup _{n \in \mathbb{N}} Z_{n}=\infty\right)=0 .
$$

No corolário a seguir encontraremos o limite superior para a probabilidade dada em (A.19). No decorrer desta demonstração, o Lema A.6 e a Proposição A.9 serão utilizados.

Corolário A.10. Seja $\left(Z_{m}\right)_{m \in \mathbb{N}}$ um martingal quadrado integrável tal que $\left|Z_{n+1}-Z_{n}\right| \leq 1$ q.c. Então, para todo $\lambda \in \mathbb{R}_{+}$,

$$
\mathbb{P}\left(\bigcup_{n=1}^{\infty}\left|Z_{n}\right|>\alpha+\frac{\exp (\lambda)-1-\lambda}{\lambda} A_{n}\right) \leq 2 \exp (-\alpha \lambda)
$$

onde $\left(A_{m}\right)_{m \in \mathbb{N}}$ denota o processo crescente associado com o submartingal $\left(Z_{m}^{2}\right)_{m \in \mathbb{N}}$ pela Decomposição de Doob.

Prova. Temos que $\left(Z_{m}\right)_{m \in \mathbb{N}}$ é um martingal quadrado integrável e satisfaz a condição $\left|Z_{n+1}-Z_{n}\right| \leq$ 1 q.c.

Assim, pelo Lema A.6, temos que a sequência $\exp \left(\lambda Z_{n}-\phi(\lambda) A_{n}\right)$ é um supermartingal positivo para todo $\lambda \in \mathbb{R}_{+}$, onde $\phi: \mathbb{R}_{+} \rightarrow \mathbb{R}_{+}$é a função definida por $\phi(\lambda)=\exp (\lambda)-1-\lambda$ e $\left(A_{m}\right)_{m \in \mathbb{N}}$ denota o processo crescente associado com o submartingal $\left(Z_{m}^{2}\right)_{m \in \mathbb{N}}$ pela Decomposição de Doob.

Pela Proposição A.9, considerando $\alpha>0$ e $\lambda>0$ números positivos arbitrários, temos que

$$
\mathbb{P}\left(\sup _{n \in N}\left(\exp \left(\lambda Z_{n}-\phi(\lambda) A_{n}\right)\right)>\exp (\lambda \alpha)\right) \leq \min \left(\frac{\exp \left(\lambda Z_{0}-\phi(\lambda) A_{0}\right)}{\exp (\lambda \alpha)}, 1\right)=\exp (-\lambda \alpha),
$$


pois $A_{0}=Z_{0}=0$. Como

$$
\begin{aligned}
\mathbb{P}\left(\sup _{n \in N}\left(\exp \left(\lambda Z_{n}-\phi(\lambda) A_{n}\right)\right)>\exp (\lambda \alpha)\right) & =\mathbb{P}\left(\bigcup_{n \in N}\left(\exp \left(\lambda Z_{n}-\phi(\lambda) A_{n}\right)>\exp (\lambda \alpha)\right)\right) \\
& =\mathbb{P}\left(\bigcup_{n \in N}\left(Z_{n}>\alpha+\lambda^{-1} \phi(\lambda) A_{n}\right)\right),
\end{aligned}
$$

então,

$$
\mathbb{P}\left(\bigcup_{n \in N}\left(Z_{n}>\alpha+\lambda^{-1} \phi(\lambda) A_{n}\right)\right) \leq \exp (-\lambda \alpha)
$$

Como $\left(Z_{m}\right)_{m \in \mathbb{N}}$ é um martingal quadrado integrável, temos que $\left(-Z_{m}\right)_{m \in \mathbb{N}}$ também é um martingal quadrado integrável. Assim,

$$
\mathbb{P}\left(\bigcup_{n \in N}\left(-Z_{n}>\alpha+\lambda^{-1} \phi(\lambda) A_{n}\right)\right) \leq \exp (-\lambda \alpha)
$$

Portanto,

$$
\mathbb{P}\left(\bigcup_{n=1}^{\infty}\left(\left|Z_{n}\right|>\alpha+\lambda^{-1} \phi(\lambda) A_{n}\right)\right)=\mathbb{P}\left(\bigcup_{n=1}^{\infty}\left(\left|Z_{n}\right|>\alpha+\frac{\exp (\lambda)-1-\lambda}{\lambda} A_{n}\right)\right) \leq 2 \exp (-\lambda \alpha)
$$

A seguir, apresentaremos o lema utilizado nos Corolários 3.9 e 3.12.

Lema A.11. Sejam $\left(H_{m}\right)_{m \in \mathbb{N}}$ uma sequência de eventos crescentes e $\left(G_{m}\right)_{m \in \mathbb{N}}$ uma sequência de eventos. Se

(i) $\mathbb{P}\left(\left(G_{n} \cap H_{n}\right) i . v.\right)=0 e$

(ii) $\mathbb{P}\left(H_{n} i . v.\right)=1$.

Então temos que

$$
\mathbb{P}\left(G_{n} \text { i.v. }\right)=0 .
$$

Prova. Por (i) temos que $\mathbb{P}\left(\left(G_{n} \cap H_{n}\right) i . v.\right)=0$ ou, equivalentemente, que

$$
\mathbb{P}\left(\bigcup_{n=1}^{\infty} \bigcap_{k=n}^{\infty}\left(G_{k}^{c} \cup H_{k}^{c}\right)\right)=1
$$

Note que a sequência de eventos $\bigcap_{k=n}^{\infty}\left(G_{k}^{c} \cup H_{k}^{c}\right)$ é crescente. Pela continuidade da probabilidade, obtemos que

$$
\lim _{n \rightarrow \infty} \mathbb{P}\left(\bigcap_{k=n}^{\infty}\left(G_{k}^{c} \cup H_{k}^{c}\right)\right)=\mathbb{P}\left(\bigcup_{n=1}^{\infty} \bigcap_{k=n}^{\infty}\left(G_{k}^{c} \cup H_{k}^{c}\right)\right)=1 .
$$

Seja $\epsilon>0$. Pela definição de limite, existe $n_{1} \in \mathbb{N}$ tal que

$$
1-\epsilon \leq \mathbb{P}\left(\bigcap_{k=n}^{\infty}\left(G_{k}^{c} \cup H_{k}^{c}\right)\right),
$$

para todo $n \geq n_{1}$. 
Agora, como $\left(H_{m}\right)_{m \in \mathbb{N}}$ é uma sequência de eventos crescentes, então $\left(H_{m}^{c}\right)_{m \in \mathbb{N}}$ é uma sequência de eventos decrescentes. Assim, temos que

$$
\bigcap_{k=n}^{\infty}\left(G_{k}^{c} \cup H_{k}^{c}\right) \subset \bigcap_{k=n}^{\infty}\left(G_{k}^{c} \cup H_{n}^{c}\right)=\left(\bigcap_{k=n}^{\infty} G_{k}^{c}\right) \cup H_{n}^{c} .
$$

De (A.22) e (A.23), para $n \geq n_{1}$ obtemos

$$
1-\epsilon \leq \mathbb{P}\left(\left(\bigcap_{k=n}^{\infty} G_{k}^{c}\right) \cup H_{n}^{c}\right) \leq \mathbb{P}\left(\bigcap_{k=n}^{\infty} G_{k}^{c}\right)+\mathbb{P}\left(H_{n}^{c}\right) .
$$

Novamente, utilizando o fato que $\left(H_{m}\right)_{m \in \mathbb{N}}$ é uma sequência de eventos crescentes, temos que

$$
\mathbb{P}\left(H_{n} \text { i.v. }\right)=\mathbb{P}\left(\bigcup_{n=1}^{\infty} H_{n}\right)=1 .
$$

Assim, para o mesmo $\epsilon$, existe $n_{2} \in \mathbb{N}$ tal que, para todo $n \geq n_{2}$,

$$
\mathbb{P}\left(H_{n}^{c}\right) \leq \frac{\epsilon}{2} .
$$

Logo, de (A.24) e (A.25),

$$
1-\frac{\epsilon}{2} \leq \mathbb{P}\left(\bigcup_{n=1}^{\infty} \bigcap_{k=n}^{\infty} G_{k}^{c}\right)
$$

Como $\epsilon$ é arbitrário, então

$$
\mathbb{P}\left(\bigcup_{n=1}^{\infty} \bigcap_{k=n}^{\infty} G_{k}^{c}\right)=1
$$

ou seja,

$$
\mathbb{P}\left(G_{n} i . v .\right)=0 .
$$


APÊNDICE A 


\section{Referências Bibliográficas}

[1] ABADI M.: Exponential approximation for hitting times in mixing processes, Math.Phys. Elec. J., Vol.7, n.2, 2001. 2

[2] ABADI, M.: Ocorrência de eventos raros em processos misturadores, Tese de Doutorado, 2001.

[3] ABADI M.: Sharp error terms and necessary conditions for exponential hitting times in mixing processes, Annals of Probability, Vol.32, p.243-264, 2004.

[4] ABADI,M.: Hitting, returning and the short correlation function, Bulletin of the Brazilian Mathematical Society, Vol.37, p.1-17, 2006. 3

[5] ABADI, M. and LAMBERT R.: The distribution of the short-return function, Nonlinearity, Vol.26, p. 1143-1162, 2013. iii, v, 2

[6] ABADI M. and SAUSSOL B.: Hitting and returning into rare events for all alpha-mixing processes, Stochastic Processes and their Applications, Vol.121, p.314, 2011.

[7] ABADI M. and VAIENTI S.: Large deviations for short recurrence, Discrete and Continuous Dynamical Systems. Series A, Vol. 21, p.729-747, 2008. iii, v, 2

[8] ABADI, M. and VERGNE N.: Sharp error terms for return time statistics under mixing condition, Journal of Theoretical Probability, Vol.22, p-18-37, 2009. 3

[9] AFRAimOviCH, V., CHAZOTteS J.R. and SAUSSOL, B.: Pointwise dimensions for Poincaré recurrence associated with maps and special flows. Discrete and Continuous Dynamical Systems, Vol.9, p.263-280, 2003. iii, v, 2, 8

[10] ASH, R.: Probability and Measure Theory. Harcourt Academic Press, Second Edition. 36

[11] BILlingSLEY, P.: Ergodic Theory and Information, first ed., New York: Wiley, 1965. 7, 8

[12] CARDENO, L., ABADI, M.: Renyi entropies and large deviations for the first match function, Preprint v, 2, 8

[13] CARDENO, L., GALlO, S., and ABADI,M.: Characterization of potential well spectrum in renewal process, preprint, 2013. iii, 3

[14] COLLET, P., GALVES A., SCHMITT B.: Repetition times for gibbsian sources, Nonlinearity, Vol.12, n.4, p.1225-1237, 1999. 2

[15] CSISZÁR, I. and SHIELDS, P.: The consistency of the BIC Markov order estimator, The Annals of Statistics, Vol.28, n.6, p.1601-1619, 2000. 3

[16] CSISZÁR, I. and TALATA, Z.: Context tree estimation for not necessarily finite memory processes, via BIC and MDL, IEEE Transactions on Information Theory, Vol.52, n.3, p.10071016, 2006. 17,18

[17] FERNÁNDEZ, R., FERRARI, P.A., GALVES, A.: Coupling, renewal and perfect simulation of chains of infinite order, Notes for the V Brazilian School of Probability, 2001. 
[18] GALVES, A. and SCHMITT, B.: Inequalities for hitting times in mixing dynamical systems, Random and Computational Dynamics, Vol.5, p.337-348, 1997.

[19] HAYDN N. and VAIENTI, S.: The limiting distribution and error terms for return time of dynamical systems, Discrete and Continuous Dynamical Systems, Vol.10, p.584-616, 2004. 2

[20] HAYDN N. and VAIENTI S.: The Rényi entropy function and the large deviation of short return times, Ergodic Theory Dynam. Systems, Vol.30, n.1, p.159-179. iii, v

[21] CSISZÁR, I.: Large-Scale Typicality of Markov Sample Paths and Consistency of MDL Order Estimators, IEEE Transactions on Information Theory, Vol.48, n.6, 2002. 3, 5

[22] HIRATA, M.: Poisson law for Axiom A diffeomorphisms, Ergodic Theory and Dynamical Systems, Vol.13, n.3, p.533-556, 1993. 2

[23] HIRATA M., SAUSSOL B. and VAIENTI S.: Statistics of return times: a general framework and new applications, Communications in Mathematical Physics, Vol.206, p.33-55, 1999. 3

[24] RADA MORA E.A.: Tempo de primeiro retorno para processos dependentes. Tese de Doutorado - Universidade de São Paulo, (in preparation). iii, v, 2

[25] NEVEU, J.: Discrete Parameter Martingales. Amsterdam, The Netherlands: North Holland, 197537,39

[26] REINERT G. and SCHBATH S.: Large Compound Poisson approximations for occurrences of multiple words, Statistics in Molecular Biology, IMS Lecture Notes - Monograph Series, Vol.33, 1999. 2

[27] REINERT G. and SCHBATH S.: Compound Poisson and Poisson process approximations for occurrences of multiple words in Markov chains, Journal of Computational Biology, Vol.5, n.2, p.223-253, 1998. 2

[28] ROQUAIN, E. and SCHBATH S.: Improved compound Poisson approximation for the number of occurrences of multiple words in a stationary Markov chain, Advances in Applied Probability, Vol.39, n.1, p.1-13, 2007. 2

[29] SAUSSOL, B., TROUBETZKOY, S. and VAIENTI, S.: Recurrence, dimensions and Lyapunov exponents, Journal of Statistical Physics, Vol.106, p. 623-634, 2002. iii, v, 2, 8

[30] SHIRYAEV, A.N.: Probability. Graduate Texts in Mathematics, Second Edition. 37, 38 